\title{
Wavelets in weighted norm spaces *
}

\author{
K. S. Kazarian, ${ }^{\dagger}$ S. S. Kazaryan ${ }^{\dagger}$ and A. San Antolín ${ }^{\S}$
}

September 25, 2018

\begin{abstract}
We give a complete characterization of the classes of weight functions for which the Haar wavelet system for $m$-dilations, $m=2,3, \ldots$ is an unconditional basis in $L^{p}(\mathbb{R}, w)$. Particulary it follows that higher rank Haar wavelets are unconditional bases in the weighted norm spaces $L^{p}(\mathbb{R}, w)$, where $w(x)=|x|^{r}, r>p-1$. These weights can have very strong zeros at the origin. Which shows that the class of weight functions for which higher rank Haar wavelets are unconditional bases is much richer than it was supposed. One of main purposes of our study is to show that weights with strong zeros should be considered if somebody is studying basis properties of a given wavelet system in a weighted norm space.
\end{abstract}

\section{Introduction}

The wavelet analysis, since its creation, has been used in many areas of applied mathematics. The main idea is as simple as to find a function (wavelet or wavelet function) defined in $\mathbb{R}$ or in $\mathbb{R}^{d}$ so that the system of its

*Math Subject Classifications. primary: 41A65; secondary: 41A25, 41A46, 46B20.

†Dept. of Mathematics, Mod. 17, Universidad Autónoma de Madrid, 28049, Madrid, Spain e-mail: kazaros.kazarian@uam.es

¥Institute of Mathematics Armenian Academy of Sciences 375019 Yerevan Armenia e-mail: kazarian@instmath.sci.am

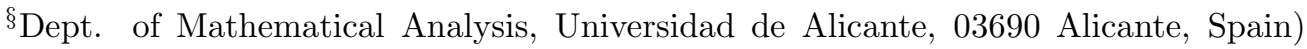
e-mail: angel.sanantolin@uam.es 
dilations and translations constitute a complete orthonormal system (ONS) in $L^{2}(\mathbb{R})$ or in $L^{2}\left(\mathbb{R}^{d}\right)$. In fact this idea was used by $\mathrm{A}$. Haar for constructing a complete ONS in $L^{2}([0,1])$ such that the expansion with respect the system of any continuous function on $[0,1]$ converges uniformly. In the univariate case the simplest dilation is the dyadic dilation. In this case a function $g \in L^{2}(\mathbb{R})$ is a wavelet if $\left\{g_{k, j}: k, j \in \mathbb{Z}\right\}$, where $g_{k, j}(x):=2^{k / 2} g\left(2^{k} x-j\right)$ is a complete ONS in the space $L^{2}(\mathbb{R})$.

If we want to study the class of weight functions $w \geq 0$ for which a given wavelet system $\left\{g_{k, j}: k, j \in \mathbb{Z}\right\}$ is a basis in a certain sense in the weighted norm space $L^{p}(\mathbb{R}, w), 1 \leq p<\infty$ then we have to consider a new phenomenon which does not arise in the case of a finite interval. In recent years several papers were published where the above question was studied. Unfortunately in the majority of those papers the phenomenon which we are going to describe is not considered. To give a preliminary idea about the main subject of our study suppose that for a given weight function $w$ there exist some functions $h$ such that

(a) $\quad \int_{\mathbb{R}} g_{k, j}(x) h(x) d x=0 \quad$ for all $\quad k, j \in \mathbb{Z}$;

(b) $\quad \frac{h}{w} \in L^{p^{\prime}}(\mathbb{R}, w), \quad 1 / p+1 / p^{\prime}=1$.

Then any non trivial function $\frac{h}{w}$ as linear continuous functional will be a non trivial element in the dual space $L^{p^{\prime}}(\mathbb{R}, w)$. Moreover, this functional vanishes on all $g_{k, j}: k, j \in \mathbb{Z}$. Hence our wavelet system is not complete in the space $L^{p}(\mathbb{R}, w)$. A necessary condition for completeness of the system $\left\{g_{k, j}\right.$ : $k, j \in \mathbb{Z}\}$ in the space $L^{p}(\mathbb{R}, w)$ is the following condition: $\frac{h}{w} \notin L^{p^{\prime}}(\mathbb{R}, w)$. Thus if we are going to describe all weight functions $w$ for which the system $\left\{g_{k, j}: k, j \in \mathbb{Z}\right\}$ is a basis in a certain sense in $L^{p}(\mathbb{R}, w)$, we have to consider all those weight functions $w$ for which the condition $(b)$ is not true. For our study we will use the technique by which similar questions were studied for incomplete systems in the weighted norm spaces (see [15, [16], 17]). We also will show that the conditions $(a)$ and $(b)$ are not hypothetical cases. We tried to produce a readable text of our study. The authors are conscious that some results of the present paper can be proved by other methods. In those cases we have opted for more classical tools with the hope to present a text which will be accessible to a wider range of readers.

Usually in concrete examples one has that the wavelet $g \in L^{1}(\mathbb{R}) \bigcap L^{2}(\mathbb{R})$. Moreover, if we are going to study the wavelet system in $L^{p}(\mathbb{R}, w)$ it will be 
natural to suppose that

$$
g \in L^{1}(\mathbb{R}) \bigcap L^{\max \left(p, p^{\prime}\right)}(\mathbb{R}) .
$$

On the other hand the purpose of the present paper is not to obtain the most general results. Hence, instead of the last restriction we will suppose that $g \in L^{1}(\mathbb{R}) \cap L^{\infty}(\mathbb{R})$. It is well known that if a wavelet $g \in L^{1}(\mathbb{R}) \bigcap L^{2}(\mathbb{R})$ then its Fourier transform $\widehat{g}$ should vanish at the origin and thus the constant functions satisfy to condition $(a)$. Which means that if someone has studied the formulated question without describing the class of functions for which the condition (a) holds and without considering weighted norm spaces $L^{p}(\mathbb{R}, w)$ with weights $w$ which does not satisfy the condition (b), then his proof is not complete. In [7](see also [6]) was given a complete characterization of weight functions $w$ for which the Haar wavelet system is an unconditional basis in $L^{p}(\mathbb{R}, w), 1 \leq p<\infty$. It was given without detailed proof because the technical details were similar with the proof given in [17. Almost at the same time was published the paper [23. In the last paper the described phenomenon was not considered. It is understandable that we will not cite all papers where similar questions have been studied.

In order to show that the question under consideration is not a technical problem which has only theoretical interest, we characterize the classes of weight functions for which the Haar wavelet system for $m$-dilations, $m=$ $2,3, \ldots$ is an unconditional basis in $L^{p}(\mathbb{R}, w)$. From the corollary of the main theorem of the last section it follows that higher rank Haar wavelets are unconditional bases in the weighted norm spaces $L^{p}(\mathbb{R}, w)$, where $w(x)=$ $|x|^{r}, r>p-1$. Which shows that the class of weight functions for which higher rank Haar wavelets are unconditional bases is much richer than it was supposed (see for example [20], where this question was studied). In Section 2 we prove an inequality for the orthogonal wavelet systems which particularly shows that in the case of general orthogonal wavelet systems the set of nontrivial functions $h$ for which the condition (a) holds is not empty. In Section 3 we give all preliminary results which will be used for our study. Next two sections are dedicated to the $m$ th rank Haar system on $[0,1]$. The results of these sections have certain interest. It should be mentioned that they are used for proofs given in the last section, where the main results for the higher rank Haar wavelets are obtained. 


\section{$2 \quad$ An inequality for wavelet type systems}

If $w \geq 0$ be a weight function on $\mathbb{R}$, i.e. a non negative locally integrable function then we write $\phi \in L^{p}(\mathbb{R}, w), 1 \leq p<\infty$ if $\phi: \mathbb{R} \rightarrow \mathbb{C}$ is measurable on $\mathbb{R}$ and the norm is defined by

$$
\|\phi\|_{L^{p}(\mathbb{R}, w)}:=\left(\int_{\mathbb{R}}|\phi(t)|^{p} w(t) d t\right)^{\frac{1}{p}}<+\infty .
$$

For a $g \in L^{2}(\mathbb{R})$ and $m=2,3, \ldots$ we will denote

$$
g_{k, j, m}(x):=m^{k / 2} g\left(m^{k} x-j\right), \quad k, j \in \mathbb{Z} .
$$

In this paper we will use a slightly modified version of the classical definition of the Fourier transform. For a function $f \in L^{1}(\mathbb{R}) \cap L^{2}(\mathbb{R})$ we put

$$
\widehat{f}(y)=\int_{\mathbb{R}} f(x) e^{-2 \pi i x y} d x .
$$

The characteristic function of a set $E$ is denoted by $\chi_{E}$ and $\mathbb{N}_{0}=\mathbb{N} \bigcup\{0\}$.

The following lemma is a well known result (cf. [4], p. 132; [12], p. 71).

Lemma 2.1. The system $\{h(\cdot-j): j \in \mathbb{Z}\}$, where $h \in L^{2}(\mathbb{R})$, is an orthonormal system if and only if

$$
\sum_{j \in \mathbb{Z}}|\widehat{h}(t+j)|^{2}=1 \quad \text { for a.e. } \quad t \in \mathbb{R} .
$$

As an obvious corollary of the above lemma we have that if $g \in L^{2}(\mathbb{R})$ is a wavelet then $|\widehat{g}(t)| \leq 1$ a.e. on $\mathbb{R}$.

Theorem 2.1. Let $g \in L^{2}(\mathbb{R})$ and $m=2,3, \ldots$ Suppose that the system $\left\{g_{k, j, m}: k \in \mathbb{N}_{0}, j \in \mathbb{Z}\right\}$ is orthonormal. Then

$$
\sum_{k=0}^{\infty}\left|\widehat{g}\left(m^{-k} x\right)\right|^{2} \leq 1
$$

Proof. It is easy to check that

$$
\widehat{g_{k, j, m}}(y)=m^{-k / 2} \widehat{g}\left(m^{-k} y\right) e^{-2 \pi i j m^{-k} y} .
$$


It is well known that for any interval $I \subset \mathbb{R},|I|=1$ the trigonometric system $\left\{e^{-2 \pi i j y}\right\}_{j \in \mathbb{Z}}$ is a complete orthonormal system in $L^{2}(I)$. Hence, for any $k \in \mathbb{Z}$ and $\Delta \subset \mathbb{R},|\Delta|=m^{k}$ the system $\left\{m^{-k / 2} e^{-2 \pi i j m^{-k} y}\right\}_{j \in \mathbb{Z}}$ will be a complete orthonormal system in $L^{2}(\Delta)$. Thus for any $f \in L^{2}(\mathbb{R})$ such that $\operatorname{supp} \widehat{f} \subseteq I,|I|=1$ we will have that

$$
S_{0}(f, x)=\sum_{j \in \mathbb{Z}} \int_{\mathbb{R}} f(t) \overline{g_{0, j, m}(t)} d t g_{0, j, m}(x)=\sum_{j \in \mathbb{Z}} \int_{I} \widehat{f}(t) \overline{\widehat{g}(t)} e^{2 \pi i j t} d t g_{0, j, m}(x) .
$$

Which yields

$$
\widehat{S_{0}(f, \cdot)}(y)=\sum_{j \in \mathbb{Z}} \int_{I} \widehat{f}(t) \overline{\widehat{g}(t)} e^{2 \pi i j t} d t \widehat{g}(y) e^{-2 \pi i j y}=\widehat{f}(y)|\widehat{g}(y)|^{2}
$$

if $y \in I$. It should be observed that the last equality holds because $\widehat{f}(\cdot) \overline{\widehat{g}(\cdot)} \in$ $L^{2}(I)$ which is true because of Lemma 2.1. If for any $k \in \mathbb{N}$ we put

$$
S_{k}(f, x)=\sum_{j \in \mathbb{Z}} \int_{\mathbb{R}} f(t) \overline{g_{k, j, m}(t)} d t g_{k, j, m}(x)
$$

in a same way we obtain that

$$
\widehat{S_{k}(f, \cdot)}(y)=\widehat{f}(y)\left|\widehat{g}\left(m^{-k} y\right)\right|^{2} \quad \text { if } \quad y \in I .
$$

By the orthogonality of the system $\left\{g_{k, j}: k \in \mathbb{N}_{0}, j \in \mathbb{Z}\right\}$ we have that

$$
S_{k}(f, \cdot) \perp S_{k^{\prime}}(f, \cdot) \quad \text { in } \quad L^{2}(\mathbb{R}) \quad \text { if } \quad k \neq k^{\prime} .
$$

Hence,

$$
\begin{gathered}
\int_{\mathbb{R}}\left|\sum_{k=0}^{l} S_{k}(f, x)\right|^{2} d x=\sum_{k=0}^{l} \int_{\mathbb{R}}\left|S_{k}(f, x)\right|^{2} d x=\sum_{k=0}^{l} \int_{\mathbb{R}}\left|\widehat{S_{k}(f, \cdot)}(y)\right|^{2} d y \\
=\int_{\mathbb{R}} \sum_{k=0}^{l}\left|\widehat{S_{k}(f, \cdot)}(y)\right|^{2} d y=\left.\left.\int_{\mathbb{R}}|\widehat{f}(y)|^{2}\left|\sum_{k=0}^{l}\right| \widehat{g}\left(m^{-k} y\right)\right|^{2}\right|^{2} d y .
\end{gathered}
$$

By (2.4) we have that

$$
\left.\left.\int_{I}|\widehat{f}(y)|^{2}\left|\sum_{k=0}^{l}\right| \widehat{g}\left(m^{-k} y\right)\right|^{2}\right|^{2} d y=\int_{I} \sum_{k=0}^{l}\left|\widehat{S_{k}(f, \cdot)}(y)\right|^{2} d y
$$




$$
\leq \int_{\mathbb{R}} \sum_{k=0}^{l}\left|\widehat{S_{k}(f, \cdot)}(y)\right|^{2} d y .
$$

By the above relations and Bessel's inequality we obtain that

$$
\begin{gathered}
\left.\left.\int_{I}|\widehat{f}(y)|^{2}\left|\sum_{k=0}^{l}\right| \widehat{g}\left(m^{-k} y\right)\right|^{2}\right|^{2} d y \leq \int_{\mathbb{R}}\left|\sum_{k=0}^{l} S_{k}(f, x)\right|^{2} d x \\
\leq\|f\|^{2}=\int_{I}|\widehat{f}(y)|^{2} d y .
\end{gathered}
$$

The last inequality can be interpreted as follows. Let

$$
\mu_{l}(y)=\sum_{k=0}^{l}\left|\widehat{g}\left(m^{-k} y\right)\right|^{2} \quad \text { if } \quad y \in I .
$$

Then for any $l \in \mathbb{N}$ the multiplicative operator $T_{l}(\phi)(y)=\mu_{l}(y) \phi(y)$ is a bounded operator $L^{2}(I) \rightarrow L^{2}(I)$ with the norm less than or equal to 1 . Which is true if and only if $\mu_{l}(y) \leq 1$.

By a simple modification of the last part, related with the application of the Bessel inequality and the proof of Theorem 2.1 we obtain the following

Theorem 2.2. Let $h^{(\nu)} \in L^{2}(\mathbb{R}), 1 \leq \nu \leq \mu$ and $m=2,3, \ldots$ Suppose that the system $\left\{h_{k, j, m}^{(\nu)}: k \in \mathbb{N}_{0}, j \in \mathbb{Z}, 1 \leq \nu \leq \mu\right\}$ is orthonormal. Then

$$
\left.\sum_{k=0}^{\infty} \sum_{\nu=1}^{\mu} \widehat{\mid h^{(\nu)}}\left(m^{-k} x\right)\right|^{2} \leq 1 .
$$

We formulate the following corollary for $g \in L^{1}(\mathbb{R}) \bigcap L^{2}(\mathbb{R})$. In the general case a similar result can be proved using the concept of points of approximate continuity (cf. [1], [2]).

Corollary 2.1. Let $g \in L^{1}(\mathbb{R}) \bigcap L^{2}(\mathbb{R})$ and $m=2,3, \ldots$ Suppose that the system $\left\{g_{k, j, m}: k \in \mathbb{N}_{0}, j \in \mathbb{Z}\right\}$ is orthonormal. Then the continuous function $\widehat{g}$ vanishes at the origin, $\widehat{g}(0)=0$.

Proof. Let $\widehat{g}(0) \neq 0$. Without loss in generality we can suppose that $\widehat{g}(0)>0$. Which yields that $\widehat{g}(y)$ is greater than $\widehat{g}(0) / 2$ in a neighborhood of the origin. The last condition contradicts to (2.2). 
By Corollary 2.1 we have that when for $g \in L^{1}(\mathbb{R}) \bigcap L^{2}(\mathbb{R})$ and $m=$ $2,3, \ldots$ the system $\left\{g_{k, j, m}: k \in \mathbb{N}_{0}, j \in \mathbb{Z}\right\}$ is orthonormal then the set of nontrivial functions $h$ defined on $\mathbb{R}$ such that

$$
(a)_{m} \quad \int_{\mathbb{R}} g_{k, j, m}(x) h(x) d x=0 \quad \text { for all } \quad k, j \in \mathbb{Z} .
$$

is not empty. Hence, having in mind that the constant function belongs to $L^{\infty}(\mathbb{R})$ we obtain

Corollary 2.2. Let $\left\{g^{(\nu)}\right\}_{\nu=1}^{\mu} \subset L^{1}(\mathbb{R}) \bigcap L^{2}(\mathbb{R})$ and $m=2,3, \ldots$ If the system $\left\{g_{k, j, m}^{(\nu)}: k \in \mathbb{Z}, j \in \mathbb{Z}, 1 \leq \nu \leq \mu\right\}$ is orthonormal then it cannot be complete in $L^{1}(\mathbb{R})$.

\section{Preliminary results}

\subsection{On $\mathcal{M}$-sets}

Further in this section we will consider that $m \geq 2$ is a fixed natural number. Let $\mathcal{M}=\mathcal{M}(m):=\left\{\left[\frac{j-1}{m^{k}}, \frac{j}{m^{k}}\right]: k \in \mathbb{Z}, j \in \mathbb{Z}\right\}$. Further, the parameter $m$ will be omitted to make the notation understandable. We will assume that any $m$-adic rational point $\xi=\frac{j}{m^{k}}, k \in \mathbb{Z}, j \in \mathbb{Z}$ is "split" into two distinct points $\xi_{l}$ and $\xi_{r}$ characterized by the following conditions: for any $-\infty<a<\xi<b<+\infty$ we have

$$
\xi_{l} \in(a, \xi], \quad \xi_{l} \notin[\xi, b), \quad \text { and } \quad \xi_{r} \in[\xi, b), \quad \xi_{r} \notin(a, \xi] .
$$

Hence, there can be easily established one to one correspondence between any $y \in \mathbb{R}$ and the sequences $\left\{\Delta_{j}(y)\right\}_{j=-\infty}^{\infty} \subset \mathcal{M}$ such that

$$
\Delta_{j+1}(y) \subset \Delta_{j}(y) \text { for all } j \in \mathbb{Z} \text { and }\left|\Delta_{j}(y)\right|=m^{-j} .
$$

When talking about the neighborhoods of the points $\xi_{l}$ and $\xi_{r}$, we will understand some intervals $(a, \xi)$ and $(\xi, b)$, respectively. The measure of the set of all $m$-adic rational points is equal to zero, hence, this assumption will not affect the results that we are going to consider. In the last section we need concept of $\mathcal{M}$-neighborhoods of $+\infty$ and $-\infty$. For $j \in \mathbb{N}_{0}$ we put

$$
\Delta_{j}(+\infty)=\mathbb{R}^{+} \backslash\left[0, m^{j}\right], \quad \text { and } \quad \Delta_{j}(-\infty)=\mathbb{R}^{-} \backslash\left[-m^{j}, 0\right],
$$

where $\mathbb{R}^{+}=[0,+\infty)$ and $\mathbb{R}^{-}=(\infty, 0]$. 


\subsubsection{Maximal function}

Let

$$
M_{\mathcal{M}} f(x)=\sup _{x \in \Delta, \Delta \in \mathcal{M}} \frac{1}{|\Delta|} \int_{\Delta}|f(t)| d t, \quad f \in L_{\text {loc }}^{1}(\mathbb{R})
$$

Let us consider also a maximal function with respect to a weight function $\omega$ defined by the following equation

$$
M_{\mathcal{M}, \omega} f(x)=\sup _{x \in \Delta, \Delta \in \mathcal{M}} \frac{1}{\omega(\Delta)} \int_{\Delta}|f(t)| \omega(t) d t, \quad f \in L_{\mathrm{loc}}^{1}(\mathbb{R}, \omega),
$$

where $\omega(\Delta)=\int_{\Delta} \omega(t) d t$.

Proposition 3.1. Let $\omega(x) \geq 0$ for $x \in \mathbb{R}, \omega \in L_{l o c}^{1}(\mathbb{R})$ and let $f \in$ $L_{\text {loc }}^{1}(\mathbb{R}, \omega)$. Then for any $\lambda>0$

$$
\omega\left(\left\{t \in \mathbb{R}: M_{\mathcal{M}, \omega} f(t)>\lambda\right\}\right) \leq \frac{1}{\lambda} \int_{\mathbb{R}}|f(t)| \omega(t) d t .
$$

Proof. If $x \in \Omega_{\lambda}(f):=\left\{t \in \mathbb{R}: M_{\mathcal{M}, \omega} f(t)>\lambda\right\}$ then for some $\Delta \in \mathcal{M}$ such that $x \in \Delta$

$$
\frac{1}{\omega(\Delta)} \int_{\Delta}|f(t)| \omega(t) d t>\lambda
$$

Observe that among all intervals which have the above properties there exists $\Delta_{x} \in \mathcal{M}$ with maximal $\omega$-measure. Thus, having in mind that $\mathcal{M}$ is numerable we can find a sequence of mutually disjoint intervals $\left\{\Delta_{\nu}\right\} \subset \mathcal{M}$ so that $\Omega_{\lambda}=\bigcup_{\nu=1}^{\infty} \Delta_{\nu}$.

We also have

Proposition 3.2. Let $\omega(x) \geq 0$ for $x \in \mathbb{R}, \omega \in L_{l o c}^{1}(\mathbb{R})$. Then for any $f \in L_{l o c}^{p}(\mathbb{R}, \omega), p>1$

$$
\int_{\mathbb{R}} M_{\mathcal{M}, \omega} f(t)^{p} \omega(t) d t \leq \frac{2^{p} p}{p-1} \int_{\mathbb{R}}|f(t)|^{p} \omega(t) d t
$$

Proof. Following the proof of the corresponding result for the Lebesgue measure (see [27], p.7) we split $f$ into two parts, $f=f_{1}+f_{2}$, where $f_{1}(x)=f(x)$ if $|f(x)| \geq \frac{\lambda}{2}$ and $f_{1}(x)=0$ otherwise. Then we have $|f(x)| \leq\left|f_{1}(x)\right|+\frac{\lambda}{2}$. 
Hence, $M_{\mathcal{M}, \omega} f(x) \leq M_{\mathcal{M}, \omega} f_{1}(x)+\frac{\lambda}{2}$ and $\Omega_{\lambda}(f) \subseteq \Omega_{\frac{\lambda}{2}}\left(f_{1}\right)$. Thus by Proposition 3.1 we have that

$$
\omega\left(\Omega_{\lambda}(f)\right) \leq \frac{2}{\lambda} \int_{\mathbb{R}}\left|f_{1}(t)\right| \omega(t) d t=\frac{2}{\lambda} \int_{\Omega_{\frac{\lambda}{2}}(f)}|f(t)| \omega(t) d t .
$$

Afterwards we have to use the following equality for any measurable function $g: \mathbb{R} \rightarrow \mathbb{R}$

$$
\begin{gathered}
\int_{\mathbb{R}}|g(t)|^{p} \omega(t) d t=p \int_{\mathbb{R}} \int_{[0,|g(t)|]} \lambda^{p-1} d \lambda \omega(t) d t \\
=p \int_{0}^{+\infty} \lambda^{p-1} \omega(\{t:|g(t)|>\lambda\}) d \lambda .
\end{gathered}
$$

Hence, by (3.5) we obtain

$$
\begin{gathered}
\int_{\mathbb{R}} M_{\mathcal{M}, \omega} f(t)^{p} \omega(t) d t \leq 2 p \int_{0}^{+\infty} \lambda^{p-2} \int_{\Omega_{\frac{\lambda}{2}}(f)}|f(t)| \omega(t) d t d \lambda= \\
2 p \int_{\mathbb{R}}|f(t)| \omega(t) \int_{0}^{2|f(t)|} \lambda^{p-2} d \lambda d t=\frac{2^{p} p}{p-1} \int_{\mathbb{R}}|f(t)|^{p} \omega(t) d t
\end{gathered}
$$

\subsubsection{Calderon-Zygmund decomposition for $m$-adic intervals}

We need a modified version for the Calderon-Zygmund decomposition (see [28]) for the $m$-adic intervals. Let $f \in L^{1}[0,1], f \geq 0$ and let $\lambda>0$ is such that

$$
\int_{[0,1]} f(t) d t<\lambda
$$

At the first step we take $m$ intervals $\left\{I_{k}\right\}_{k=1}^{m} \subset \mathcal{M} \bigcap[0,1]$ such that $\left|I_{k}\right|=$ $\frac{1}{m}, 1 \leq k \leq m$ and $\bigcup_{k=1}^{m} I_{k}=[0,1]$. Let $\left\{I_{k_{l}}\right\}_{l=1}^{m_{1}} \subset\left\{I_{k}\right\}_{k=1}^{m}$ be all those intervals for which $\eta_{I_{k_{l}}}>\lambda, 1 \leq l \leq m_{1}<m$, where

$$
\frac{1}{|I|} \int_{I} f(t) d t:=\eta_{I}
$$

Those intervals are renamed $G_{1}, \ldots, G_{m_{1}}$. Clearly,

$$
\lambda<\frac{1}{\left|G_{l}\right|} \int_{G_{l}} f(t) d t \leq m \lambda .
$$


If $\eta_{I_{k}} \leq \lambda$ for all $1 \leq k \leq m$ then we put $m_{1}=0$. On the next step we repeat the same procedure on any of those intervals that were not renamed. The collection of all $m$-adic intervals which are separated on the second step are renamed $G_{m_{1}+1}, \ldots, G_{m_{2}}$. For those intervals the condition (3.6) holds again. On the $\nu$ th step all $m$-adic intervals which are separated are renamed $G_{m_{\nu-1}+1}, \ldots, G_{m_{\nu}}$. If no any interval is separated then we put $m_{\nu+1}=m_{\nu}$. This procedure produces a collection of disjoint $m$-adic intervals $\left\{G_{l}\right\}$ for which the condition (3.6) holds and

$$
|\Omega|=\sum_{l}\left|G_{l}\right|<\frac{1}{\lambda} \sum_{l} \int_{G_{l}} f(t) d t \leq \frac{1}{\lambda} \int_{[0,1]} f(t) d t,
$$

where $\Omega=\bigcup_{l} G_{l}$ and for any $I \in \mathcal{M} \bigcap[0,1], I \subset \Omega^{c}:=[0,1] \backslash \Omega$ we have that $\eta_{I} \leq \lambda$. The collection $\left\{G_{l}\right\}$ will be called Calderon-Zygmund $m$-adic decomposition at level $\lambda$. Let

$$
g(x)=f(x) \chi_{\Omega^{c}}(x)+\sum_{l} \eta_{G_{l}} \chi_{G_{l}}(x) \quad \text { and } \quad b(x)=f(x)-g(x) .
$$

We skip the details of the proof of the following

Proposition 3.3. Let $f \in L^{1}[0,1]$, and let $\lambda>0$ be such that $\int_{[0,1]}|f(t)| d t<$ $\lambda$. Then there exists a family of disjoint sets $\left\{G_{l}\right\}_{l \in \Upsilon} \subset \mathcal{M}$ such that

$$
|f(x)| \leq \lambda \quad \text { a.e. on } \Omega^{c} \text {, where } \Omega=\bigcup_{l \in \Upsilon} G_{l} \text {, }
$$

(3.7) is true and for any $l \in \Upsilon$ holds (3.6). Moreover, $f(x)=g(x)+b(x)$, where $g$ is defined by (3.8) and the following conditions hold:

$$
\begin{gathered}
|g(x)| \leq m \lambda \quad \text { a.e. on } \quad[0,1] \\
\|g(x)\|_{p}^{p} \leq(m \lambda)^{p-1}\|f\|_{1} \quad \text { for all } \quad 1 \leq p<\infty \\
\int_{G_{l}} b(t) d t=0 \quad \text { for all } \quad l \in \Upsilon .
\end{gathered}
$$

For dyadic intervals a similar result was obtained by C. Watari [29]. 


\subsubsection{Classes of $\mathcal{M}_{p}, p \geq 1$ weights}

Definition 3.1. We say that a non negative locally integrable function $\omega$ satisfies the condition $\mathcal{M}_{p}, p \geq 1$ if

$$
\omega(\Delta)\left[\int_{\Delta} \omega^{-\frac{1}{p-1}}(t) d t\right]^{p-1} \leq C_{p}|\Delta|^{p} \quad \forall \Delta \in \mathcal{M},
$$

where $C_{p}>0$ is independent of $\Delta \in \mathcal{M}$. For $p=1$ it is understood that $\left[\int_{\Delta} \omega^{-\frac{1}{p-1}}(t) d t\right]^{p-1}:=\left\|\omega^{-1}\right\|_{L^{\infty}(\Delta)}$.

We say that $\omega$ satisfies the condition $\mathcal{M}_{p}(G)$, where $G \subset \mathbb{R}$ if (3.13) holds for all $\Delta \in \mathcal{M} \cap G$. The reader should observe that the conditions $\mathcal{M}_{p}([0,1])$ and $\mathcal{M}_{p}((0,1])$ are distinct. In the second case the intervals $\left[0,2^{-j}\right], j \in \mathbb{N}$ should be excluded when one checks the inequality (3.13).

The following lemma is obvious.

Lemma 3.1. Let $\omega$ satisfies the condition $\mathcal{M}_{p}, p>1$ then $\psi=\omega^{-\frac{1}{p-1}}$ satisfies the condition $\mathcal{M}_{p^{\prime}}$, where $\frac{1}{p}+\frac{1}{p^{\prime}}=1$.

We follow the ideas given in [3] to prove the following result.

Proposition 3.4. Let $\omega(x) \geq 0$ for $x \in \mathbb{R}, \omega \in L_{\text {loc }}^{1}(\mathbb{R})$. Then for any $f \in L_{l o c}^{p}(\mathbb{R}, \omega), p>1$

$$
\int_{\mathbb{R}} M_{\mathcal{M}} f(t)^{p} \omega(t) d t \leq B_{p} \int_{\mathbb{R}}|f(t)|^{p} \omega(t) d t,
$$

for some $B_{p}>0$ independent of $f$ if and only if $\omega$ satisfies the condition $\mathcal{M}_{p}$. Proof. Suppose that (3.14) is true. For any $\Delta \in \mathcal{M}$ we have by (3.1) that

$$
\frac{1}{|\Delta|} \int_{\Delta}|f(t)| d t \chi_{\Delta}(x) \leq M_{\mathcal{M}} f(x) .
$$

Hence, by (3.14) we have that

$$
\left(\frac{1}{|\Delta|} \int_{\Delta}|f(t)| d t\right)^{p} \omega(\Delta) \leq B_{p} \int_{\mathbb{R}}|f(t)|^{p} \omega(t) d t .
$$

Letting $f(t)=\omega^{-\frac{1}{p-1}}(t) \chi_{\Delta}(t)$ we obtain (3.13) with $C_{p}=B_{p}$. To prove the opposite assertion one has to use Proposition 3.1 and the following 
Lemma 3.2. Let $\omega$ satisfies the condition $\mathcal{M}_{p}, p>1$ then there exists $\varepsilon>0$ such that $\omega$ satisfies the condition $\mathcal{M}_{p-\varepsilon}$.

We skip the rest of the proof because the proof in [3] works with small changes.

Definition 3.2. We say that a non negative locally integrable function $\omega$ satisfies the condition $\mathcal{M}_{\infty}$ if there exists $C>0$ and $\delta>0$ such that for any $\Delta \in \mathcal{M}$ and any measurable subset $E \subseteq \Delta$

$$
\frac{\omega(E)}{\omega(\Delta)} \leq C\left(\frac{|E|}{|\Delta|}\right)^{\delta}
$$

We skip the detailed proofs of the following two lemmas because the corresponding proofs in [3] for $A_{p}$ weights work with obvious changes.

Lemma 3.3. Let $\omega$ satisfies the condition $\mathcal{M}_{p}, p>1$ then there exist $r>0$ and $C>0$ such that

$$
\left(\frac{1}{|\Delta|} \int_{\Delta} \omega(x)^{1+r}\right)^{\frac{1}{1+r}} \leq C \frac{\omega(\Delta)}{|\Delta|} \quad \forall \Delta \in \mathcal{M} .
$$

Lemma 3.4. Let $\omega$ satisfies the condition $\mathcal{M}_{p}$ for some $p>1$ then $\omega$ satisfies the condition $\mathcal{M}_{\infty}$.

We need also the following

Lemma 3.5. Let $\omega$ satisfies the condition $\mathcal{M}_{p}$ for some $p>1$ then

$$
\omega \notin L\left(\Delta_{j}(+\infty)\right), \quad \omega \notin L\left(\Delta_{j}(-\infty)\right), \quad \forall j \in \mathbb{N}_{0} .
$$

Proof. We prove the assertion for the neighborhoods of $+\infty$. For this purpose we observe that there exists $C_{p}>0$ such that for any $j \in \mathbb{N}_{0}$

$$
\omega\left(\Delta_{-j-1}\right) \leq C_{p} \omega\left(\Delta_{-j-1} \backslash \Delta_{-j}\right) .
$$

For any $j \in \mathbb{N}_{0}$ and any locally integrable function $f \geq 0$ we have that $M_{\mathcal{M}} f(x) \geq m^{-j} \int_{\left[0, m^{j}\right]} f(t) d t$ if $x \in\left[0, m^{j}\right]$. By Proposition 3.4 we obtain that

$$
\left(m^{-j} \int_{\left[0, m^{j}\right]} f(t) d t\right)^{p} \omega\left(\left[0, m^{j}\right]\right) \leq B_{p} \int_{\left[0, m^{j}\right]} f(t)^{p} \omega(t) d t .
$$


Putting $j+1$ instead of $j$ in the above inequality and letting $f$ be the characteristic function of the set $\Delta_{-j-1} \backslash \Delta_{-j}$ we obtain the inequality (3.17). If $\omega \in L\left(\Delta_{j_{0}}(+\infty)\right)$ then for any $\varepsilon>0$ there exists $N \in \mathbb{N}$ such that

$$
\int_{\left[m^{N},+\infty\right)} \omega(t) d t<\varepsilon .
$$

Which leads to a contradiction with the condition (3.17). Evidently the proof for the neighborhoods of $-\infty$ is similar.

Definition 3.3. Let $\omega \geq 0$ be a weight function defined on $\mathbb{R}^{+}$. We will say that $\omega$ satisfies the condition $\mathcal{M}_{p}^{y}\left(\mathbb{R}^{+}\right), p \geq 1$ for some $y \in \mathbb{R}^{+}$if

$$
\omega\left(\Delta_{j}(y)\right)\left[\int_{\mathbb{R}^{+} \backslash \Delta_{j}(y)} \omega^{-\frac{1}{p-1}}(t) d t\right]^{p-1} \leq C_{p}\left|\Delta_{j}(y)\right|^{p} \quad \forall j \in \mathbb{Z},
$$

where $C_{p}>0$ is independent of $j \in \mathbb{Z}$.

We will not formulate the definition of the condition $\mathcal{M}_{p}^{y}\left(\mathbb{R}^{-}\right)$because it is clear from the context.

Definition 3.4. Let $\omega \geq 0$ be a weight function defined on $\Delta$, where $\Delta \in \mathcal{M}$, $|\Delta|=m^{l}$. We will say that $\omega$ satisfies the condition $\mathcal{M}_{p}^{y}(\Delta), p \geq 1$ for some $y \in \Delta$ if

$$
\omega\left(\Delta_{j}(y)\right)\left[\int_{\Delta \backslash \Delta_{j}(y)} \omega^{-\frac{1}{p-1}}(t) d t\right]^{p-1} \leq C_{p}\left|\Delta_{j}(y)\right|^{p} \quad \forall j>l,
$$

where $C_{p}>0$ is independent of $j$.

Lemma 3.6. Let $w \geq 0$ be a weight function defined on $[0,1]$ such that $w$ satisfies the condition $\mathcal{M}_{p}^{y}([0,1])$ for some $y \in[0,1]$ and $1<p<\infty$. Then there exists $q_{p}>1$ such that

$$
\int_{[0,1] \backslash \Delta_{j+1}(y)} w^{-\frac{1}{p-1}}(t) d t\left(\int_{[0,1] \backslash \Delta_{j}(y)} w^{-\frac{1}{p-1}}(t) d t\right)^{-1} \geq q_{p}
$$

for all $j \in \mathbb{N}$. 
Proof. For any $j \in \mathbb{N}$ we have that

$$
\begin{gathered}
\int_{[0,1] \backslash \Delta_{j+1}(y)} w^{-\frac{1}{p-1}}(t) d t\left(\int_{[0,1] \backslash \Delta_{j}(y)} w^{-\frac{1}{p-1}}(t) d t\right)^{-1} \\
\geq 1+\int_{\Delta_{j}(y) \backslash \Delta_{j+1}(y)} w^{-\frac{1}{p-1}}(t) d t\left(\int_{[0,1] \backslash \Delta_{j}(y)} w^{-\frac{1}{p-1}}(t) d t\right)^{-1} \\
\geq 1+\int_{\Delta_{j}(y) \backslash \Delta_{j+1}(y)} w^{-\frac{1}{p-1}}(t) d t\left(w\left(\Delta_{j}(y)\right)\right)^{\frac{1}{p-1}} C_{p}^{-\frac{1}{p-1}}\left|\Delta_{j}(y)\right|^{-\frac{p}{p-1}} \\
\geq 1+\left|\Delta_{j}(y) \backslash \Delta_{j+1}(y)\right|^{\frac{p}{p-1}} C_{p}^{-\frac{1}{p-1}}\left|\Delta_{j}(y)\right|^{-\frac{p}{p-1}} \geq 1+C_{p}^{-\frac{1}{p-1}}\left(\frac{m-1}{m}\right)^{\frac{p}{p-1}} .
\end{gathered}
$$

Putting $q_{p}=1+C_{p}^{-\frac{1}{p-1}}\left(\frac{m-1}{m}\right)^{\frac{p}{p-1}}$ we finish the proof.

Following three lemmas will be used in the last section. In the proofs we will use the following notation: $a E=\{a t: t \in E\}$.

Lemma 3.7. Let $\omega \geq 0$ satisfies the condition $\mathcal{M}_{p}\left(\mathbb{R}^{+}\right), p>1$ with a constant $C_{p}>0$. Then for any $N \in \mathbb{N}$ the weight function $\omega_{N}(x):=\omega\left(m^{N} x\right)$ satisfies the condition $\mathcal{M}_{p}([0,1])$ with the same constant $C_{p}$.

Proof. For any $E \in \mathcal{M} \bigcap[0,1]$ we have

$$
\begin{aligned}
\omega_{N}(E)\left[\int_{E} \omega_{N}^{-\frac{1}{p-1}}(t) d t\right]^{p-1} & =m^{-N} \int_{m^{N} E} \omega(x) d x\left[m^{-N} \int_{m^{N} E} \omega^{-\frac{1}{p-1}}(x) d x\right]^{p-1} \\
& \leq m^{-p N} C_{p}\left|m^{N} E\right|^{p}=C_{p}|E|^{p}
\end{aligned}
$$

We have used that $m^{N} E \in \mathcal{M}$.

Lemma 3.8. Let $N \in \mathbb{N}$ and let $y \in\left[0, m^{N}\right]$. Suppose that $\omega \geq 0$ satisfies the condition $\mathcal{M}_{p}\left(\left[0, m^{N}\right] \backslash\{y\}\right), p>1$ with a constant $C_{p}>0$. Then the weight function $\omega_{N}(x):=\omega\left(m^{N} x\right)$ satisfies the condition $\mathcal{M}_{p}\left([0,1] \backslash\left\{y_{N}\right\}\right)$ with the same constant $C_{p}$, where $y_{N}=m^{-N} y$.

Proof. For any $E \in \mathcal{M} \cap\left([0,1] \backslash\left\{y_{N}\right\}\right)$ we observe that the interval $m^{N} E \in$ $\mathcal{M} \cap\left(\left[0, m^{N}\right] \backslash\{y\}\right)$. The rest of the proof is the same as above. 
Lemma 3.9. Let $N \in \mathbb{N}$ and let $y \in\left[0, m^{N}\right]$. Suppose that $\omega \geq 0$ satisfies the condition $\mathcal{M}_{p}^{y}\left(\left[0, m^{N}\right]\right), p>1$ with a constant $C_{p}>0$. Then the weight function $\omega_{N}(x):=\omega\left(m^{N} x\right)$ satisfies the condition $\mathcal{M}_{p}^{y_{N}}([0,1])$ with the same constant $C_{p}$, where $y_{N}=m^{-N} y$.

Proof. For any $j \in \mathbb{N}$ we have

$$
\begin{aligned}
& \omega_{N}\left(\Delta_{j}\left(y_{N}\right)\right)\left[\int_{[0,1] \backslash \Delta_{j}\left(y_{N}\right)} \omega_{N}^{-\frac{1}{p-1}}(t) d t\right]^{p-1}=m^{-N} \int_{\Delta_{j-N}(y)} \omega(x) d x \\
& \times\left[m^{-N} \int_{\left[0, m^{N}\right] \backslash \Delta_{j-N}(y)} \omega^{-\frac{1}{p-1}}(x) d x\right]^{p-1} \\
& \leq m^{-p N} C_{p}\left|\Delta_{j-N}(y)\right|^{p}=C_{p}\left|\Delta_{j}(y)\right|^{p} .
\end{aligned}
$$

\subsection{Higher rank Haar wavelets}

We bring the definition of higher rank Haar wavelets without recalling the general theory of multiresolution analysis. For relations of these type of wavelets with $p$-adic analysis see [21]. Let $\varphi(x)=\chi_{[0,1]}(x)$ and let

$$
V(m)=\operatorname{span}\left\{\varphi_{1, j, m}(x): 0 \leq j \leq m-1\right\}
$$

for any $m=2,3, \ldots$. Afterwards, let $\left\{h^{(\nu)}(x): 0 \leq \nu \leq m-1\right\}$ be an orthonormal basis in $V(m)$ such that $h^{(0)}(x)=\varphi(x)$. The system

$$
H(m)=\left\{h_{k, j, m}^{(\nu)}(x): k \in \mathbb{Z} ; j \in \mathbb{Z} ; 1 \leq \nu \leq m-1\right\}
$$

where

$$
h_{k, j, m}^{(\nu)}(x)=m^{k / 2} h^{(\nu)}\left(m^{k} x-j\right)
$$

will be called $m$ th rank Haar system. Sometimes we will use also the following notation

$$
h_{\Delta}^{(\nu)}:=h_{k, j, m}^{(\nu)}(x) \quad \text { when } \quad \Delta=\left[\frac{j}{m^{k}}, \frac{j+1}{m^{k}}\right] .
$$

The orthogonality of the system (3.21) is obvious.

Theorem 3.1. The system $H(m)$ is complete in $L^{p}(\mathbb{R}), 1<p<\infty$. 
Proof. Let $p, 1<p<\infty$ be fixed. It is easy to observe that the proof will be finished if we show that for any $\varphi_{1, j, m}(x), 0 \leq j \leq m-1$ and any $\varepsilon>0$ there exists a finite linear combination $P_{j}$ of functions $\left\{h_{k, l, m}^{(\nu)}(x): k \in \mathbb{Z} \backslash \mathbb{N}_{0} ; l \in \mathbb{Z} ; 1 \leq \nu \leq m-1\right\}$ such that $\left\|\varphi_{1, j, m}-P_{j}\right\|_{L^{p}(\mathbb{R})}<\varepsilon$. Let $l \in \mathbb{N}$ be such that $m^{1 / 2} m^{l(1 / p-1)}<\varepsilon$. We set

$$
V^{(l)}(m)=\operatorname{span}\left\{\varphi_{1, j, m}(x): 0 \leq j \leq m^{l+1}-1\right\} .
$$

It is clear that $\operatorname{dim} V^{(l)}(m)=m^{l+1}$. Let us show by induction that there are exactly $m^{l+1}-1$ functions from the system $\left\{h_{k, j, m}^{(\nu)}(x): 1 \geq k \geq-l+1 ; j \in \mathbb{Z} ; 1 \leq \nu \leq m-1\right\}$ with supports in $\left[0, m^{l}\right]$.

If $l=0$ then it is obvious. Suppose that for some $\mu \in \mathbb{N}$ we have that the number of functions from the system

$$
\left\{h_{k, j, m}^{(\nu)}(x): 1 \geq k \geq-\mu+1 ; j \in \mathbb{Z} ; 1 \leq \nu \leq m-1\right\}
$$

with supports in $\left[0, m^{\mu}\right]$ is equal to $m^{\mu+1}-1$. Then it is clear that there are $\left(m^{\mu+1}-1\right) m$ functions from the system (3.24) that have their supports in $\left[0, m^{\mu+1}\right]$. Note that the functions $\left\{h_{-\mu, 0, m}^{(\nu)}(x): 1 \leq \nu \leq m-1\right\}$ vanish outside the closed interval $\left[0, m^{\mu+1}\right]$. Thus we have $\left(m^{\mu+1}-1\right) m+m-1=$ $m^{\mu+2}-1$ mutually orthogonal functions in (3.24) which have their supports in $\left[0, m^{\mu+1}\right]$.

Let $\left\{g_{i}\right\}_{i=1}^{m^{l+1}-1}$ be all functions from the system

$$
\left\{h_{k, j, m}^{(\nu)}(x): 1 \geq k \geq-l+1 ; j \in \mathbb{Z} ; 1 \leq \nu \leq m-1\right\}
$$

that have their supports in $\left[0, m^{l}\right]$. Evidently $\left\{g_{i}\right\}_{i=1}^{m^{l+1}-1} \subset V^{(l)}(m)$.

Let $g_{0}(x)=\chi_{\left[0, m^{l}\right]}(x)$. Then $g_{0} \in V^{(l)}(m)$ and $g_{0}$ is orthogonal to all elements of $\left\{g_{i}\right\}_{i=1}^{m^{l+1}-1}$. Hence, $\left\{g_{i}\right\}_{i=0}^{m^{l+1}-1}$ is a basis in $V^{(l)}(m)$ and

$$
\varphi_{1, j, m}=\sum_{i=0}^{m^{l+1}-1} a_{i}^{(j)} g_{i}, \quad \text { where } \quad a_{0}^{(j)}=m^{-l} \int_{\left[0, m^{l}\right]} \varphi_{1, j, m}(t) d t .
$$

Thus we obtain that for any $0 \leq j \leq m-1$

$$
\left\|\varphi_{1, j, m}-\sum_{i=1}^{m^{l+1}-1} a_{i}^{(j)} g_{i}\right\|_{L^{p}(\mathbb{R})}=\left\|a_{0}^{(j)} g_{0}\right\|_{L^{p}(\mathbb{R})}=m^{1 / 2} m^{l(1 / p-1)}<\varepsilon .
$$




\section{$4 \quad m$ th rank Haar system on $[0,1]$}

Let $\mathrm{h}_{0}(x) \equiv 1$ for $x \in[0,1]$. For any $n \in \mathbb{N}$ we have a unique representation

$$
n=m_{k}+j-1, \quad \text { where } \quad k \in \mathbb{N}, 1 \leq j \leq m^{k},
$$

and

$$
m_{k}=1+m+m^{2}+\cdots+m^{k-1}, \quad m_{1}=1 .
$$

For any $1 \leq \nu \leq m-1$ we put

$$
\mathrm{h}_{n}^{(\nu)}(x)=h_{k, j-1, m}^{(\nu)}(x) \quad \text { for } x \in[0,1] .
$$

Afterwards we enumerate the functions in the following way

$$
\begin{aligned}
& \mathrm{h}_{l}(x)=h^{(l)}(x) \quad \text { for } \quad 1 \leq l \leq m-1 ; \\
& \mathrm{h}_{l}(x)=\mathrm{h}_{n}^{(\nu)}(x) \quad \text { for } \quad l=\nu+n(m-1), n \in \mathbb{N} .
\end{aligned}
$$

We denote the $m$ th rank Haar system by $\mathcal{H}(m)=\left\{\mathrm{h}_{l}(x)\right\}_{l=0}^{\infty}$. We also let

$$
\mu_{0}=0, \mu_{1}=\mu_{0}+m-1, \cdots, \mu_{k+1}=\mu_{k}+(m-1) m^{k}, \cdots .
$$

The following lemma is the analogue of Schauder's lemma for the classical Haar system (see [26]). For any $f \in L^{1}[0,1]$ and for any $1 \leq j \leq m^{k}, k \in \mathbb{N}$ we put

$$
\Theta_{\mu_{k}+j(m-1)}(f, x)=\sum_{l=0}^{\mu_{k}} a_{l}(f) \mathrm{h}_{l}(x)+\sum_{s=0}^{j-1} \sum_{\nu=1}^{m-1} a_{k, s, m}^{(\nu)}(f) h_{k, s, m}^{(\nu)}(x),
$$

where

$$
a_{l}(f)=\int_{[0,1]} f(t) \mathrm{h}_{l}(t) d t ; \quad a_{k, s, m}^{(\nu)}(f)=\int_{[0,1]} f(t) h_{k, s, m}^{(\nu)}(t) d t .
$$

Lemma 4.1. Let $f \in L^{1}[0,1]$ and let $1 \leq j \leq m^{k}, k \in \mathbb{N}_{0}$. Then the partial sum $\Theta_{\mu_{k}+j(m-1)}(f, x)$ is constant on any interval from the collection of sets

$$
\begin{gathered}
\left\{\left[\frac{s}{m^{k+1}}, \frac{s+1}{m^{k+1}}\right]: 0 \leq s \leq j m-1\right\}, \\
\left\{\left[\frac{l}{m^{k}}, \frac{l+1}{m^{k}}\right]: j \leq l \leq m^{k}-1\right\} .
\end{gathered}
$$

Moreover, for any $\Delta$ from (4.6) or from (4.7)

$$
\Theta_{\mu_{k}+j(m-1)}(f, x)=\frac{1}{|\Delta|} \int_{\Delta} f(t) d t \quad \text { for } \quad x \in \Delta .
$$


Proof. At first we show that the assertion of the lemma is true for $\Theta_{\mu_{k}}(f, x)$, $k \in \mathbb{N}_{0}$. Indeed,

$$
\operatorname{span}\left\{\mathrm{h}_{l}, 0 \leq l \leq m^{k}-1\right\}=V(m),
$$

where $V(m)$ is defined by (3.20). Hence, for any $\Delta$ from (4.7) with $j=1$ we have that for $x \in \Delta$

$$
\Theta_{\mu_{k}}(f, x)=\sum_{l=0}^{m^{k}-1} \int_{[0,1]} f(t) \varphi_{k, l, m}(t) d t \varphi_{k, l, m}(x)=\frac{1}{|\Delta|} \int_{\Delta} f(t) d t .
$$

Afterwards we observe that in the general case

$$
\Theta_{\mu_{k}+j(m-1)}(f, x)=\Theta_{\mu_{k+1}}(f, x) \quad \text { if } \quad x \in\left[0, \frac{j}{m^{k}}\right]
$$

and

$$
\Theta_{\mu_{k}+j(m-1)}(f, x)=\Theta_{\mu_{k}}(f, x) \quad \text { if } \quad x \in\left[\frac{j}{m^{k}}, 1\right] .
$$

Which finishes the proof.

By Lemma 4.1 we obtain the following corollaries.

Corollary 4.1. For any $m=2,3, \ldots$ the system $\mathcal{H}(m)$ is a basis in any space $L^{p}[0,1], 1 \leq p<\infty$.

Proof. By Lemma 4.1 as in the case of the classical Haar system we have that

$$
\left\|\Theta_{\mu_{k}+j(m-1)}\right\|_{L^{p} \rightarrow L^{p}} \leq 1 \quad \text { for all } \quad 1 \leq j \leq m^{k}, k \in \mathbb{N} .
$$

To finish the proof we have to check that $\lim _{l \rightarrow \infty}\left|a_{l}(f)\right|\left\|\mathrm{h}_{l}\right\|_{L^{p}[0,1]}=0$. We skip the technical details because afterwards we are going to return to the similar question in the weighted norm case.

Corollary 4.2. For any $f \in L^{1}[0,1]$ the Fourier series of $f$ with respect to the system $\mathcal{H}(m), m=2,3, \ldots$ converges almost everywhere to $f$ on $[0,1]$.

Proof. For every $x \in[0,1]$ which is a Lebesgue point of $f$ we have that

$$
\lim _{k \rightarrow \infty} \Theta_{\mu_{k}}(f, x)=f(x) .
$$

Afterwards we observe that $\left|a_{l}(f)\right|\left|\mathrm{h}_{l}(x)\right| \leq C M_{\mathcal{M}}(f, x)$ which finishes the proof. 
For any $k \in \mathbb{N}_{0}$ and any $1 \leq j \leq m^{k}$ consider the kernel

$$
K_{k j}(t, x)=\sum_{l=0}^{\mu_{k}} \mathrm{~h}_{l}(t) \mathrm{h}_{l}(x)+\sum_{s=0}^{j-1} \sum_{\nu=1}^{m-1} h_{k, s, m}^{(\nu)}(t) h_{k, s, m}^{(\nu)}(x) .
$$

Let $\left\{G_{i}: 1 \leq i \leq m^{k}+j(m-1)\right\}$ be mutually disjoint sets from (4.6) and (4.7). Further in the paper we will need the following result.

Lemma 4.2. Let $k \in \mathbb{N}_{0}$ and $1 \leq j \leq m^{k}$. Then the kernel

$$
K_{k j}(t, x)=\frac{1}{\left|G_{i}\right|} \quad \text { for } \quad(t, x) \in G_{i}^{2}, 1 \leq i \leq m^{k}+j(m-1) ;
$$

and

$$
K_{k j}(t, x)=0, \quad \text { if } \quad(t, x) \in[0,1]^{2} \backslash \bigcup_{i=1}^{m^{k}+j(m-1)} G_{i}^{2} .
$$

Proof. We have that $\left\{\left|G_{i}\right|^{-1 / 2} \chi_{G_{i}}(x)\right\}_{i=1}^{m^{k}+j(m-1)}$ is an orthonormal system of functions. From Lemma 4.1 it follows that the orthonormal system of functions $\left\{\mathrm{h}_{l}(x)\right\}_{l=0}^{\mu_{k}} \bigcup\left\{h_{k, s, m}^{(\nu)}(x): 0 \leq s \leq j-1,1 \leq \nu \leq m-1\right\}$ can be obtained from the set of functions $\left\{\left|G_{i}\right|^{-1 / 2} \chi_{G_{i}}(x)\right\}_{i=1}^{m^{k}+j(m-1)}$ by an orthogonal transformation. Hence,

$$
\begin{aligned}
K_{k j}(t, x) & =\sum_{i=1}^{m^{k}+j(m-1)}\left|G_{i}\right|^{-1 / 2} \chi_{G_{i}}(t)\left|G_{i}\right|^{-1 / 2} \chi_{G_{i}}(x) \\
& =\sum_{i=1}^{m^{k}+j(m-1)}\left|G_{i}\right|^{-1} \chi_{G_{i}}(t) \chi_{G_{i}}(x) .
\end{aligned}
$$

Definition 4.1. We say that a system of functions $\left\{\phi_{k}\right\}_{k=1}^{\infty} \subset L^{\infty}[0,1]$ is total with respect to $L^{1}[0,1]$ if

$$
\int_{[0,1]} f(t) \phi_{k}(t) d t=0 \quad \text { for all } \quad k \in \mathbb{N} \quad \text { for some } \quad f \in L^{1}[0,1]
$$

if and only if $f=0$ a.e. on $[0,1]$. 
By Lemma 4.1 it follows immediately

Corollary 4.3. The system $\mathcal{H}(m), m=2,3, \ldots$ is total with respect to $L^{1}[0,1]$.

Theorem 4.1. For any $m=2,3, \ldots$ the system $\mathcal{H}(m)$ is an unconditional basis in any space $L^{p}[0,1], 1<p<\infty$.

The reader can find well known facts about unconditional bases in [24]. For any sequence $\epsilon=\left\{\epsilon_{l}\right\}_{l=0}^{\infty}$, where $\epsilon= \pm 1$ we consider an operator $I_{\epsilon}$ : $L^{1}[0,1] \rightarrow L^{0}[0,1]$ defined as follows $I_{\epsilon}(f, x)=\sum_{l=0}^{\infty} \epsilon_{l} a_{l}(f) \mathrm{h}_{l}$.

Proposition 4.1. The operator $I_{\epsilon}$ is of weak- $(1,1)$ type.

Proof. We adopt the idea of the proof given in [29]. Let $f \in L^{\infty}[0,1]$ and suppose that $\lambda>\|f\|_{1}$. Without loss in generality we can suppose that $f \geq 0$ (see [27], pp. 21-22). By Proposition [3.3 we write $f(x)=g(x)+$ $b(x)$, where $g$ satisfies the condition (3.10). The system $\mathcal{H}(m)$ is a complete orthonormal system. Hence, $I_{\epsilon}: L^{2}[0,1] \rightarrow L^{2}[0,1]$ is an isometry. Thus by the Tchebychev inequality we will have

$$
\left|\left\{x \in[0,1]:\left|I_{\epsilon}(g, x)\right|>\lambda\right\}\right| \leq \frac{1}{\lambda^{2}} \int_{[0,1]} g^{2}(x) d x \leq \frac{m}{\lambda}\|g\|_{1} \leq \frac{m}{\lambda}\|f\|_{1},
$$

where the last inequality follows by (3.11).Afterwards, we apply the following property of $m$-adic intervals. If $\Delta_{1}, \Delta_{2} \subset \mathcal{M}$ then only two relations are possible or $\Delta_{1} \bigcap \Delta_{2}=\emptyset$ or one of those intervals is a subset of another interval. By the definition of the system $\mathcal{H}(m)$ and by (3.12) it is easy to deduce that $I_{\epsilon}(b, x)=0$ for $x \in \Omega^{c}$. Thus by (3.7) and (4.12) we obtain

$$
\left|\left\{x \in[0,1]:\left|I_{\epsilon}(f, x)\right|>\lambda\right\}\right| \leq \frac{m+1}{\lambda}\|f\|_{1} .
$$

From the last inequality readily follows that for any $f \in L^{1}[0,1]$ the series $\sum_{l=0}^{\infty} \epsilon_{l} a_{l}(f) \mathrm{h}_{l}$ converges in measure on $[0,1]$. Observe that in the proof of the inequality (4.13) the condition $f \in L^{\infty}[0,1]$ was used only to claim the existence of $I_{\epsilon}(f, x)$. Hence, the proof is complete.

The analogue of Proposition 4.1 for the Haar system was obtained by S. Yano [31. 
Proof. By Proposition 4.1 and the Marcinkiewicz interpolation theorem (see [32]) we obtain that $\mathcal{H}(m)$ is an unconditional basis in $L^{p}[0,1], 1<p \leq 2$. Afterwards by duality we finish the proof of Theorem 4.1 .

For the system $\mathcal{H}(m)$ we put

$$
G_{m}(f, x)=\left(\sum_{l=0}^{\infty}\left|a_{l}(f) \mathrm{h}_{l}(x)\right|^{2}\right)^{\frac{1}{2}}, \quad \text { where } \quad f \in L^{1}[0,1] .
$$

For the operator $G_{m}: L^{1}[0,1] \rightarrow L^{0}[0,1]$ the following proposition holds.

Proposition 4.2. The operator $G_{m}$ is of weak- $(1,1)$ type.

Proof. Let $f=\sum_{l=0}^{N} a_{l} \mathrm{~h}_{l}$ be any polynomial with respect to the system $\mathcal{H}(m)$ and let $\epsilon=\left\{\epsilon_{l}\right\}_{l=0}^{N}$ be any Rademacher sequence. By a well known inequality (see [14],p.8) we have that for any $0<\alpha<1$ and and any $x \in[0,1]$

$$
\mathcal{P}\left(I_{\epsilon}(f, x)>\alpha G_{m}(f, x)\right)>\frac{1}{3}(1-\alpha)^{2} .
$$

Observe that $G_{m}\left(I_{\epsilon}(f, \cdot), x\right)=G_{m}(f, x)$ for any Rademacher sequence $\epsilon$. For any $\lambda>0$ we have that if the following two events $\left\{I_{\epsilon}(f, x)>\alpha G_{m}(f, x)\right\}$, $\left\{G_{m}(f, x)>\frac{\lambda}{\alpha}\right\}$ then $\left\{I_{\epsilon}(f, x)>\lambda\right\}$. Hence, by Proposition 4.1 we finish the proof for the polynomials with respect to the system $\mathcal{H}(m)$.

For arbitrary $f \in L^{1}[0,1]$ we have that the sequence $G_{m}\left(\Theta_{n}(f, \cdot), x\right)$ is an increasing sequence which a.e. converges to $G_{m}(f, x)$. Hence,

$$
\begin{gathered}
\left|\left\{G_{m}(f, x)>\lambda\right\}\right|=\lim _{n \rightarrow+\infty}\left|\left\{G_{m}\left(\Theta_{n}(f, \cdot), x\right)>\lambda\right\}\right| \\
<\frac{C}{\lambda} \sup _{n}\left\|\Theta_{n}(f, \cdot)\right\|_{L^{1}[0,1]} \leq \frac{C_{1}}{\lambda}\|f\|_{L^{1}[0,1]}
\end{gathered}
$$

By standard arguments (see [30]) one can derive from Theorem 4.1 that for all $1<p<\infty$

$$
\left\|G_{m}(f, \cdot)\right\|_{L^{p}[0,1]} \simeq\|f\|_{L^{p}[0,1]} .
$$




\subsection{Haar wavelet systems as unconditional bases in $L^{p}(\mathbb{R}), 1<p<\infty$.}

From Theorem 4.1 we easily derive

Theorem 4.2. For any $m=2,3, \ldots$ the system $H(m)$ is an unconditional basis in any space $L^{p}(\mathbb{R}), 1<p<\infty$.

Further we will use the following notations: $\mathbb{R}^{+}=[0,+\infty), \mathbb{R}^{-}=(-\infty, 0]$ and $\mathbb{Z}^{+}=\mathbb{R}^{+} \cap \mathbb{Z}$ having in mind the agreement introduced in Section 3.1 . For technical reasons we divide the system $H(m)$ into two parts:

$$
\begin{array}{r}
H^{+}(m)=\left\{h_{k, j, m}^{(\nu)}(x): k \in \mathbb{Z} ; j \geq 0 ; 1 \leq \nu \leq m-1\right\}, \\
H^{-}(m)=\left\{h_{k, j, m}^{(\nu)}(x): k \in \mathbb{Z} ; j \leq-1 ; 1 \leq \nu \leq m-1\right\} .
\end{array}
$$

We are going to show that the systems $H^{+}(m), H^{-}(m)$ are unconditional bases respectively in the spaces $L^{p}\left(\mathbb{R}^{+}\right)$and $L^{p}\left(\mathbb{R}^{-}\right), 1<p<\infty$. Let us prove the following

Theorem 4.3. For any $m=2,3, \ldots$ the system $H^{+}(m)$ is an unconditional basis in any space $L^{p}\left(\mathbb{R}^{+}\right), 1<p<\infty$.

Proof. Let $f \in L^{p}\left(\mathbb{R}^{+}\right)$and let $\Omega \subset \mathbb{Z} \times \mathbb{Z}^{+}$be a finite set. For any $1 \leq \nu \leq$ $m-1$ consider the sum

$$
S_{\Omega}^{(\nu)}(f, x)=\sum_{(k, j) \in \Omega} c_{k j}^{(\nu)}(f) h_{k, j, m}^{(\nu)}(x),
$$

where

$$
c_{k j}^{(\nu)}(f)=\int_{\mathbb{R}^{+}} f(t) h_{k, j, m}^{(\nu)}(t) d t .
$$

Let $N \in \mathbb{N}$ be such that for all $(k, j) \in \Omega h_{k, j, m}^{(\nu)}(x)=0$ if $x \in\left[m^{N},+\infty\right)$. Consider the dilation operator $D_{N}(\phi)(x)=m^{\frac{N}{2}} \phi\left(m^{N} x\right)$. It is clear that $D_{N}\left(h_{k, j, m}^{(\nu)}\right) \in \mathcal{H}(m)$ for any $h_{k, j, m}^{(\nu)}$ which satisfies to the above conditions if we consider the restriction on $[0,1]$ of the image of the operator. Thus

$$
D_{N}\left(S_{\Omega}^{(\nu)}(f, \cdot)\right)(x)=\sum_{(k, j) \in \Omega} c_{k j}^{(\nu)}(f) D_{N}\left(h_{k, j, m}^{(\nu)}\right)(x)
$$


on $[0,1]$ is a finite linear combination of elements from $\mathcal{H}(m)$. We also have that if $(k, j) \in \Omega$

$$
c_{k j}^{(\nu)}(f)=\int_{\left[0, m^{N}\right]} f(t) h_{k, j, m}^{(\nu)}(t) d t=\int_{[0,1]} D_{N}(f)(t) D_{N}\left(h_{k, j, m}^{(\nu)}\right)(t) d t .
$$

Hence, $D_{N}\left(S_{\Omega}^{(\nu)}(f, \cdot)\right)(x)$ on $[0,1]$ coincides with the sum of a subsequence of the expansion of the function $D_{N}(f)$ with respect to the system $\mathcal{H}(m)$. By Theorem 4.2 we obtain that there exists $C_{p}>0$ which depends only on $p$ such that

$$
\int_{[0,1]}\left|D_{N}\left(S_{\Omega}^{(\nu)}(f, \cdot)\right)(t)\right|^{p} d t \leq C_{p}^{p} \int_{[0,1]}\left|D_{N}(f)(t)\right|^{p} d t
$$

which yields

$$
\left\|S_{\Omega}^{(\nu)}(f, \cdot)\right\|_{L^{p}\left(\mathbb{R}^{+}\right)} \leq C_{p}\|f\|_{L^{p}\left(\mathbb{R}^{+}\right)} .
$$

It is clear that in a similar way we can check that $H^{-}(m)$ is an unconditional basis in any space $L^{p}\left(\mathbb{R}^{-}\right), 1<p<\infty$. Thus we the proof of Theorem 4.2 is finished.

\section{2 $m$ th rank Haar system in $L^{p}([0,1], w), 1 \leq p<\infty$.}

Theorem 4.4. For any $m=2,3, \ldots$ the system $\mathcal{H}(m)$ is a basis in the weighted norm space $L^{p}([0,1], w), 1 \leq p<\infty$ if and only if $w$ satisfies the condition $\mathcal{M}_{p}([0,1])$.

Proof. By Corollary 4.3 we easily obtain that the system $\mathcal{H}(m)$ is complete in $L^{p}([0,1], w), 1 \leq p<\infty$. Suppose that $w$ satisfies the condition $\mathcal{M}_{p}([0,1])$. Then it is evident that

$$
\frac{\chi_{[0,1]}}{w} \in L^{p^{\prime}}([0,1], w), \quad \text { where } \quad \frac{1}{p}+\frac{1}{p^{\prime}}=1
$$

Hence, the system $\mathcal{H}(m)=\left\{\mathrm{h}_{l}(x)\right\}_{l=0}^{\infty}$ is minimal in $L^{p}([0,1], w)$ and its conjugate system is the system $\mathcal{H}^{*}(m)=\left\{\frac{1}{w(x)} \mathrm{h}_{l}(x)\right\}_{l=0}^{\infty}$. Thus for any $f \in$ 
$L^{p}([0,1], w)$, the coefficients of its expansion with respect to the system $\mathcal{H}(m)$ are equal to

$$
b_{l}(f)=\int_{[0,1]} f(t) \frac{1}{w(t)} \mathrm{h}_{l}(t) w(t) d t=\int_{[0,1]} f(t) \mathbf{h}_{l}(t) d t=a_{l}(f) .
$$

Hence, for any $k \in \mathbb{N}$ and $1 \leq j \leq m^{k}$ the partial sums of the mentioned expansion with indices $\mu_{k}+j(m-1)$ coincide with $\Theta_{\mu_{k}+j(m-1)}(f, x)$ (see subsection 4). By Lemma 4.1 it follows easily that

$$
\left\|\Theta_{\mu_{k}+j(m-1)}(f, \cdot)\right\|_{L^{p}([0,1], w)} \leq C\|f\|_{L^{p}([0,1], w)},
$$

where $C=C(w, p, m)$ is independent of $f$. If we prove that

$$
\lim _{l \rightarrow+\infty}\left|b_{l}(f)\right|\left\|\mathrm{h}_{l}\right\|_{L^{p}([0,1], w)}=0
$$

then it will follow that (4.17) holds for all $n \in \mathbb{N}$. Which yields that the system $\mathcal{H}(m)$ is a basis $L^{p}([0,1], w)$. We have that $b_{l}(f)=\int_{[0,1]}[f(t)-P(t)] \mathrm{h}_{l}(t) d t$ for any $P(t)=\sum_{k=0}^{l-1} d_{k} \mathrm{~h}_{k}(t)$. If $\mathrm{h}_{l}(x)=h_{k, j, m}^{(\nu)}(x)$ for $x \in[0,1]$ and $\Delta=$ $\left[\frac{j}{m^{k}}, \frac{j+1}{m^{k}}\right]$ then

$$
\begin{gathered}
\left|b_{l}(f)\right|\left\|\mathrm{h}_{l}\right\|_{L^{p}([0,1], w)} \leq \\
\leq\|f-P\|_{L^{p}([0,1], w)}\left\|\frac{h_{k, j, m}^{(\nu)}}{w^{\frac{1}{p}}}\right\|_{L^{p^{\prime}(\Delta)}}\left\|h^{(\nu)}\right\|_{L^{\infty}([0,1])} m^{\frac{k}{2}}[w(\Delta)]^{\frac{1}{p}} \\
\leq\|f-P\|_{L^{p}([0,1], w)}\left\|h^{(\nu)}\right\|_{L^{\infty}([0,1])}^{2} m^{k}[w(\Delta)]^{\frac{1}{p}}\left[\int_{\Delta} w^{-\frac{1}{p-1}}\right]^{\frac{1}{p^{\prime}}} \\
\leq\|f-P\|_{L^{p}([0,1], w)}\left\|h^{(\nu)}\right\|_{L^{\infty}([0,1])}^{2} C_{p}^{\frac{1}{p}} .
\end{gathered}
$$

The last inequality yields (4.18) because the system $\mathcal{H}(m)$ is complete in $L^{p}([0,1], w)$.

To prove the necessity suppose that the system $\mathcal{H}(m)$ is a basis in the weighted norm space $L^{p}([0,1], w)$, where $1 \leq p<\infty$.

Let $\mathcal{H}^{*}(m)=\left\{\mathrm{h}_{l}^{*}(x)\right\}_{l=0}^{\infty}$ be the conjugate system of the basis $\mathcal{H}(m)$. Then we have that

$$
\int_{[0,1]}\left[\mathrm{h}_{0}^{*}(t) w(t)-1\right] \mathrm{h}_{l}(t) d t=0 \quad \text { for all } \quad l \in \mathbb{N}_{0} .
$$


Hence, $\mathbf{h}_{0}^{*}(t)=\frac{1}{w(t)} \in L^{p^{\prime}}([0,1], w)$. Thus we obtain that

$$
\mathrm{h}_{l}^{*}(x)=\frac{\mathrm{h}_{l}(x)}{w(x)} \quad \text { for all } \quad l \in \mathbb{N}_{0}
$$

Thus for any $f \in L^{p}([0,1], w) n$th partial sums of its expansion with respect to the basis $\mathcal{H}(m)$ coincide with $\Theta_{n}(f, x)$. By Lemma 4.1 it follows that for some $C \geq 1$ such that for any $\Delta \in \mathcal{M}$

$$
\left.\sup \frac{1}{|\Delta|^{p} \mid} \int_{\Delta} f(t) d t\right|^{p} w(\Delta) \leq C^{p},
$$

where the supremum is taken over all $\|f\|_{L^{p}([0,1], w)} \leq 1$. The last inequality easily yields (3.13) with $C_{p}=C^{p}$.

The prove of the following result technically is much more complicated. The main line of our proof is close to the one given in [10] (see also [3] and [17], [5]).

Theorem 4.5. For any $m=2,3, \ldots$ the system $\mathcal{H}(m)$ is an unconditional basis in the weighted norm space $L^{p}([0,1], w), 1<p<\infty$ if and only if $w$ satisfies the condition $\mathcal{M}_{p}([0,1])$.

Lemma 4.3. Suppose that $w$ is a weight function which satisfies the condition $\mathcal{M}_{\infty}([0,1])$. Then for any $\lambda>0$, any $0<\gamma<1$ and for any $f \in L^{1}[0,1]$

$$
\begin{aligned}
w\left(\left\{x \in[0,1]: G_{m}(f, x)>2 \lambda \quad \text { and } \quad M_{\mathcal{M}} f(x) \leq \gamma \lambda\right\}\right) \\
\leq C \gamma^{\delta} w\left(\left\{x \in[0,1]: G_{m}(f, x)>\lambda\right\}\right)
\end{aligned}
$$

where $C>0$ is independent of $f, \lambda>0$ and $\gamma>0$, while $\delta>0$ is the corresponding constant from Definition 3.2.

In the formulation of the following assertion we use the agreement formulated in the Section 3.1.

Lemma 4.4. For any $f \in L^{1}[0,1]$ and any $\lambda>0$ there exists a finite or denumerable set of disjoint closed intervals $\left\{\Delta_{k}\right\}_{k \in \Upsilon}$ such that the set

$$
E_{\lambda}(f)=\left\{x \in[0,1]: G_{m}(f, x)>\lambda\right\}=\bigcup_{k \in \Upsilon} \Delta_{k}
$$


Proof. According to our agreement for any $x \in[0,1]$ there exists a unique sequence of closed intervals $\Gamma_{k}(x) \subset \mathcal{M}$ such that $\Gamma_{k}(x) \subset \Gamma_{k-1}(x)$ for all $k \in \mathbb{N}$ and $\left|\Gamma_{k}(x)\right|=m^{-k}, \bigcap_{k=0}^{\infty} \Gamma_{k}(x)=x$. For any $x_{0} \in E_{\lambda}(f)$ there exists $k\left(x_{0}\right) \in \mathbb{N}_{0}$ so that $\Gamma_{k\left(x_{0}\right)}\left(x_{0}\right) \subseteq E_{\lambda}(f)$ and $\Gamma_{k\left(x_{0}\right)-1}\left(x_{0}\right)$ at least contains a point which does not belong to $E_{\lambda}(f)$. Indeed, if $G_{m}\left(f, x_{0}\right)>\lambda$ then there exists $N \in \mathbb{N}$ such that

$$
\left[G_{m}\left(f, x_{0}\right)\right]^{2} \geq \sum_{l=0}^{N}\left|a_{l}(f) \mathrm{h}_{l}\left(x_{0}\right)\right|^{2}>\lambda^{2} .
$$

Hence, for some $\nu \in \mathbb{N}$ the sum $\sum_{l=0}^{N}\left|a_{l}(f) \mathrm{h}_{l}\left(x_{0}\right)\right|^{2}$ is constant on $\Gamma_{\nu}\left(x_{0}\right)$. Which means that $\Gamma_{\nu}\left(x_{0}\right) \subseteq E_{\lambda}(f)$. The number $k\left(x_{0}\right)$ will be the smallest index for which the last relation holds. Afterwards, one observes that $\max _{x \in E_{\lambda}(f)}\left|\Gamma_{k(x)}(x)\right|:=\mu_{0}$ exists. There exist only finitely many disjoint intervals in the set $\left\{\Gamma_{k(x)}(x): x \in E_{\lambda}(f)\right\}$ with length equal to $\mu_{0}$. Let $\Delta_{j}\left(1 \leq j \leq n_{1}\right)$ be all such intervals. Let $E_{2}=E_{\lambda}(f) \backslash\left(\bigcup_{j=1}^{n_{1}} \Delta_{j}\right)$ and repeat the same procedure taking $E_{2}$ instead of $E_{\lambda}(f)$. Thus step by step we construct the finite or denumerable set of disjoint closed intervals $\left\{\Delta_{k}\right\}_{k \in \Upsilon}$ which satisfy the conditions of lemma.

Proof of Lemma 4.3. Let $\Delta_{l}$ be an arbitrary closed interval from (4.19). At the first step we have to prove the following relation

$$
\mid\left\{x \in \Delta_{l}: G_{m}(f, x)>2 \lambda \quad \text { and } \quad M_{\mathcal{M}} f(x) \leq \gamma \lambda\right\}|\leq C \gamma| \Delta_{l} \mid,
$$

where $C>0$ is independent of $f, \lambda, \gamma$ and $\Delta_{l}$. Suppose that there is at least a point $y_{l} \in \Delta_{l}$ such that $M_{\mathcal{M}} f\left(y_{l}\right) \leq \gamma \lambda$. Otherwise there is nothing to prove. Let $\Delta_{l}^{*} \in \mathcal{M}$ be the interval which satisfies the following conditions: $\Delta_{l}^{*} \supset \Delta_{l},\left|\Delta_{l}^{*}\right|=m\left|\Delta_{l}\right|$. Let $f(x)=f_{1}(x)+f_{2}(x)$, where

$$
f_{1}(x)=\left(f(x)-f_{\Delta_{l}^{*}}\right) \chi_{\Delta_{l}^{*}}(x), \quad f_{\Delta}=\frac{1}{|\Delta|} \int_{\Delta} f(t) d t, \Delta \in \mathcal{M}
$$

and $f_{2}(x)=f(x)-f_{1}(x)$. By Proposition 4.2 we have that

$$
\begin{aligned}
\mid\left\{G_{m}\left(f_{1}, x\right)\right. & \left.>\frac{\lambda}{2}\right\}\left|\leq \frac{2 C_{1}}{\lambda}\left\|f_{1}\right\|_{L^{1}[0,1]}=\frac{4 C_{1}}{\lambda} \int_{\Delta_{l}^{*}}\right| f(t) \mid d t \\
& \leq \frac{4 m C_{1}}{\lambda}\left|\Delta_{l}\right| M_{\mathcal{M}} f\left(y_{l}\right) \leq 4 m C_{1} \gamma\left|\Delta_{l}\right| .
\end{aligned}
$$


On the other hand we have that $\left|\Delta_{l}^{*}\right|=m^{-\kappa}$ for some $\kappa \in \mathbb{N}_{0}$. Thus for all $0 \leq$ $l \leq \mu_{\kappa}$ we have that $a_{l}(f)=a_{l}\left(f_{2}\right)$, which yields $G_{m}\left(f_{2}, x\right)=G_{m}\left(\Theta_{\mu_{\kappa}}(f, \cdot), x\right)$ if $x \in \Delta_{l}^{*}$. There exists at least one point $z_{l} \in \Delta_{l}^{*}$ such that $G_{m}\left(f, z_{l}\right) \leq \lambda$. Thus if $x \in \Delta_{l}^{*}$ then

$$
G_{m}\left(f_{2}, x\right)=G_{m}\left(\Theta_{\mu_{\kappa}}(f, \cdot), x\right) \leq G_{m}\left(f, z_{l}\right) \leq \lambda .
$$

We have that if $x \in \Delta_{l}^{*}$ then

$$
G_{m}(f, x) \leq G_{m}\left(f_{1}, x\right)+G_{m}\left(f_{2}, x\right) \leq G_{m}\left(f_{1}, x\right)+\lambda .
$$

Hence, by (4.21) we finish the proof of (4.20).

We have that the weight function $w$ satisfies the condition $\mathcal{M}_{\infty}([0,1])$. Thus we obtain that

$$
w\left(\left\{x \in \Delta_{l}: G_{m}(f, x)>2 \lambda \text { and } M_{\mathcal{M}} f(x) \leq \gamma \lambda\right\}\right) \leq C \gamma^{\delta} w\left(\Delta_{l}\right)
$$

where $C>0$ is independent of $f, \lambda>0, \gamma>0$ and $\Delta_{l}$. Hence, by Lemma 4.19 we finish the proof.

Proof of Theorem 4.5. The necessity follows from Theorem 4.4. Suppose that $w$ satisfies the condition $\mathcal{M}_{p}([0,1])$. By Lemma 4.3 we derive

$$
\begin{aligned}
\int_{[0,1]} G_{m}^{p}(f, x) w(x) d x= & p 2^{p} \int_{0}^{+\infty} \lambda^{p-1} w\left(\left\{x \in[0,1]: G_{m}(f, x)>2 \lambda\right\}\right) d \lambda \\
& \leq K_{p} \int_{0}^{+\infty} \lambda^{p-1} w\left(\left\{x \in[0,1]: M_{\mathcal{M}} f(x)>\gamma \lambda\right\}\right) d \lambda \\
& +K_{p} C \gamma^{\delta} \int_{0}^{+\infty} \lambda^{p-1} w\left(\left\{x \in[0,1]: G_{m}(f, x)>\lambda\right\}\right) d \lambda
\end{aligned}
$$

Let $\gamma_{0}>0$ be such that $K_{p} C \gamma_{0}^{\delta}<\frac{1}{2}$. Then we obtain that

$$
\int_{[0,1]} G_{m}^{p}(f, x) w(x) d x \leq 2 K_{p} \gamma_{0}^{-p} \int_{[0,1]} M_{\mathcal{M}}^{p} f(x) w(x) d x .
$$

By Proposition 3.4 we finish the proof. 


\section{$5 \quad$ The system $\mathcal{H}_{0}(m)=\left\{\mathbf{h}_{l}(x)\right\}_{l=1}^{\infty}$ in $L^{p}([0,1], w)$}

In this section we will use the following result (see [15]-[18])

Theorem 5.1. Let $\left\{f_{n}\right\}_{n=1}^{\infty} \subseteq L^{\infty}(E)$ be an orthonormal system of realvalued functions defined on a measurable set $E, 0<|E|<+\infty$ and suppose that $\left\{f_{n}\right\}_{n=1}^{\infty}$ is total with respect to $L^{1}(E)$. Let, furthermore, $N \in \mathbb{N}$ and $w \in L^{1}(E)$ be a weight function. For the system $\left\{f_{n}\right\}_{n=N+1}^{\infty}$ to be closed and/or minimal it is necessary and sufficient that the following conditions 1) and/or 2), respectively, are satisfied:

1) any function of the form $(w)^{-1} \sum_{n=1}^{N} c_{n} f_{n}$, where $c_{n}(1 \leq n \leq N)$ are real numbers, belongs to $L^{p^{\prime}}(E, w)$ if and only if every $c_{n}$ is zero;

2) for every $k(k=N+1, N+2, \ldots)$ there exist uniquely determined real numbers $b_{n}^{(k)}(1 \leq n \leq N)$ such that the function

$$
g_{k}=\frac{1}{w}\left[\sum_{n=1}^{N} b_{n}^{(k)} f_{n}+f_{k}\right]
$$

belongs to $L^{p^{\prime}}(E, w) \quad\left(\frac{1}{p}+\frac{1}{p^{\prime}}=1\right)$.

The following two lemmas are easy consequences of Theorem 5.1. We skip the details of the proofs because they are similar to the case of the Haar system [19].

Lemma 5.1. For any $m=2,3, \ldots$ the system $\mathcal{H}_{0}(m)$ is complete in a weighted norm space $L^{p}([0,1], w), 1 \leq p<\infty$ if and only if there exists at least one point $y \in[0,1]$ such that

$$
\frac{1}{w} \notin L^{\frac{1}{p-1}}\left(\Delta_{j}(y)\right) \quad \text { for all } \quad j \in \mathbb{N} .
$$

Lemma 5.2. For any $m=2,3, \ldots$ the system $\mathcal{H}_{0}(m)$ is minimal in a weighted norm space $L^{p}([0,1], w), 1 \leq p<\infty$ if and only if $\frac{1}{w} \in L^{1}([0,1])$ or for a point $y \in[0,1]$

$$
\frac{1}{w} \in L^{\frac{1}{p-1}}\left([0,1] \backslash \Delta_{j}(y)\right) \quad \text { for all } j \in \mathbb{N} .
$$

By Lemmas 5.1 and 5.2 it follows easily 
Lemma 5.3. For any $m=2,3, \ldots$ the system $\mathcal{H}_{0}(m)$ is complete and minimal in a weighted norm space $L^{p}([0,1], w), 1 \leq p<\infty$ if and only if there exists only one point $y \in[0,1]$ such that the conditions (5.1), (5.2) hold.

Further in this section we will suppose that the weight function $w$ satisfies the conditions (5.1) and (5.2). Hence, the system $\mathcal{H}_{0}(m)$ is complete and minimal in the weighted norm space $L^{p}([0,1], w), 1 \leq p<\infty$ with the unique conjugate system $\mathcal{H}_{0}^{*}(m)$. By Theorem 5.1 applied for our case it is easy to see that the system $\mathcal{H}_{0}^{*}(m)=\left\{\mathrm{h}_{l}^{*}(x)\right\}_{l=1}^{\infty}$ is defined by the following equations:

$$
\mathrm{h}_{l}^{*}(x)=\frac{\mathrm{h}_{l}(x)-\mathrm{h}_{l}(y)}{w(x)} .
$$

For any $f \in L^{p}([0,1], w)$ and for any $1 \leq j \leq m^{k}, k \in \mathbb{N}$ we put

$$
\Theta_{\mu_{k}+j(m-1)}^{(0)}(f, x)=\sum_{l=1}^{\mu_{k}} c_{l}(f) \mathrm{h}_{l}(x)+\sum_{s=0}^{j-1} \sum_{\nu=1}^{m-1} c_{k, s, m}^{(\nu)}(f) h_{k, s, m}^{(\nu)}(x),
$$

where

$$
c_{l}(f)=\int_{[0,1]} f(t) \mathrm{h}_{l}^{*}(t) d t ; \quad c_{k, s, m}^{(\nu)}(f)=\int_{[0,1]} f(t)\left[h_{k, s, m}^{(\nu)}(t)-h_{k, s, m}^{(\nu)}(y)\right] d t .
$$

Let $\Delta_{k j}(y)$ be the interval from the collection of sets (4.6), (4.7) such that $y \in \Delta_{k j}(y)$.

Lemma 5.4. For any $f \in L^{p}([0,1], w)$ and for any $1 \leq j \leq m^{k}, k \in \mathbb{N}$ we have that $\Theta_{\mu_{k}+j(m-1)}^{(0)}(f, x)$ is constant on any interval from the collection of sets (4.6), (4.7). Moreover,

$$
\Theta_{\mu_{k}+j(m-1)}^{(0)}(f, x)=-\frac{1}{\left|\Delta_{k j}(y)\right|} \int_{[0,1] \backslash \Delta_{k j}(y)} f(t) d t \quad \text { for } \quad x \in \Delta_{k j}(y),
$$

and for any $\Delta$ from (4.6) or from (4.7) which does not coincide with $\Delta_{k j}(y)$

$$
\Theta_{\mu_{k}+j(m-1)}^{(0)}(f, x)=\frac{1}{|\Delta|} \int_{\Delta} f(t) d t \quad \text { for } \quad x \in \Delta .
$$

Proof. In the proof we use the notation of Lemma 4.2. Let $\Delta$ be any interval from the collection of sets (4.6), 4.7) such that $\Delta \cap \Delta_{k j}(y)=\emptyset$. Then we have that

$$
\Theta_{\mu_{k}+j(m-1)}^{(0)}(f, x)=\int_{[0,1]} f(t)\left[\tilde{K}_{k j}(t, x)-\tilde{K}_{k j}(y, x)\right] d t
$$




$$
=\sum_{i=1}^{m^{k}+j(m-1)} \int_{G_{i}} f(t)\left[K_{k j}(t, x)-K_{k j}(y, x)\right] d t,
$$

where $\tilde{K}_{k j}(t, x)=K_{k j}(t, x)-1$, hence, $\tilde{K}_{k j}(t, x)-\tilde{K}_{k j}(y, x)=K_{k j}(t, x)-$ $K_{k j}(y, x)$. Suppose that $G_{\nu}=\Delta_{k j}(y)$ and take any $i_{0} \neq \nu, 1 \leq i_{0} \leq m^{k}+$ $j(m-1)$. Then by Lemma 4.2 we obtain that for $x \in G_{i_{0}}$ we obtain that

$$
\begin{gathered}
\sum_{i=1}^{m^{k}+j(m-1)} \int_{G_{i}} f(t)\left[K_{k j}(t, x)-K_{k j}(y, x)\right] d t=\int_{G_{i_{0}}} f(t) K_{k j}(t, x) d t \\
=\frac{1}{\left|G_{i_{0}}\right|} \int_{G_{i_{0}}} f(t) d t .
\end{gathered}
$$

On the other hand if $x \in G_{\nu}$ by Lemma 4.2 we will have that

$$
\begin{gathered}
\Theta_{\mu_{k}+j(m-1)}^{(0)}(f, x)=\sum_{i=1, i \neq \nu}^{m^{k}+j(m-1)} \int_{G_{i}} f(t)\left[K_{k j}(t, x)-K_{k j}(y, x)\right] d t \\
=-\frac{1}{\left|G_{\nu}\right|} \sum_{i=1, i \neq \nu}^{m^{k}+j(m-1)} \int_{G_{i}} f(t) d t=-\frac{1}{\left|G_{\nu}\right|} \int_{[0,1] \backslash G_{\nu}} f(t) d t .
\end{gathered}
$$

Lemma 3.6 and Lemma 5.3 easily yield

Lemma 5.5. Let $w \geq 0$ be a weight function defined on $[0,1]$ such that $w$ satisfies the condition $\mathcal{M}_{p}^{y}([0,1])$ for some $y \in[0,1]$ and $1<p<\infty$. Then the conditions (5.1), (5.2) hold and the system $\mathcal{H}_{0}(m)$ is complete and minimal in a weighted norm space $L^{p}([0,1], w)$.

Theorem 5.2. For any $m=2,3, \ldots$ the system $\mathcal{H}_{0}(m)$ is a basis in the weighted norm space $L^{p}([0,1], w), 1<p<\infty$ if and only if there exists a point $y \in[0,1]$ such that $w$ satisfies the conditions $\mathcal{M}_{p}([0,1] \backslash\{y\})$ and $\mathcal{M}_{p}^{y}([0,1])$.

Proof. Necessity. If the system $\mathcal{H}_{0}(m)$ is a basis in the weighted norm space $L^{p}([0,1], w)$ then it is a complete minimal system in $L^{p}([0,1], w)$. Then we 
will have that for any $f \in L^{p}([0,1], w)$ and for any $1 \leq j \leq m^{k}, k \in \mathbb{N}$ the partial sum operators are uniformly bounded

$$
\sup _{1 \leq j \leq m^{k}, k \in \mathbb{N}}\left\|\Theta_{\mu_{k}+j(m-1)}^{(0)}\right\|_{L^{p}([0,1], w) \rightarrow L^{p}([0,1], w)}:=M_{0} \leq B_{p},
$$

where $B_{p}>0$. We have that

$$
M_{0} \geq \max _{1 \leq i \leq m^{k}+j(m-1)} \sup _{\|f\|_{L^{p}\left(G_{i}, w\right)} \leq 1}\left\|\Theta_{\mu_{k}+j(m-1)}^{(0)}\right\|_{L^{p}([0,1], w)}
$$

If $G_{i} \bigcap \Delta_{k j}(y)=\emptyset$ then by Lemma 5.4 we will have that

$$
\begin{gathered}
\sup _{\|f\|_{L^{p}\left(G_{i}, w\right)} \leq 1}\left\|\Theta_{\mu_{k}+j(m-1)}^{(0)}\right\|_{L^{p}\left(G_{i}, w\right)}=\frac{1}{\left|G_{i}\right|} \sup _{\|f\|_{L^{p}\left(G_{i}, w\right)} \leq 1}\left|\int_{G_{i}} f(t) d t\right| w\left(G_{i}\right)^{\frac{1}{p}} \\
=\left|G_{i}\right|^{-1} w\left(G_{i}\right)^{\frac{1}{p}}\left\|w^{-\frac{1}{p}}\right\|_{L^{p^{\prime}}\left(G_{i}\right)} .
\end{gathered}
$$

If $G_{\nu}=\Delta_{k j}(y)$ then in the same way as above we obtain that

$$
\left|\Delta_{k j}(y)\right|^{-p} w\left(\Delta_{k j}(y)\right)\left(\int_{\Delta_{k j}(y)} w^{-\frac{1}{p-1}} d t\right)^{p-1} \leq B_{p}^{p} .
$$

Sufficiency. By Lemma 5.5 we have that the system $\mathcal{H}_{0}(m)$ is complete and minimal in a weighted norm space $L^{p}([0,1], w)$. Hence, by Lemma 5.4 we obtain that for any $f \in L^{p}([0,1], w)$ and for any $1 \leq j \leq m^{k}, k \in \mathbb{N}$

$$
\begin{gathered}
\int_{[0,1]}\left|\Theta_{\mu_{k}+j(m-1)}^{(0)}(f, t)\right|^{p} w(t) d t=\sum_{\substack{1 \leq i \leq m^{k}+j(m-1) \\
i \neq \nu}}\left|\frac{1}{\left|G_{i}\right|} \int_{G_{i}} f(t) d t\right|^{p} \int_{G_{i}} w(t) d t \\
\quad+\left|\frac{1}{\left|G_{\nu}\right|} \int_{[0,1] \backslash G_{\nu}} f(t) d t\right|^{p} \int_{G_{\nu}} w(t) d t \leq B_{p}^{p} \int_{[0,1]}|f(t)|^{p} w(t) d t .
\end{gathered}
$$

The last inequality follows because $w$ satisfies the conditions $\mathcal{M}_{p}([0,1] \backslash\{y\})$ and $\mathcal{M}_{p}^{y}([0,1])$. To finish the proof we have to show that $\lim _{l \rightarrow \infty}\left|c_{l}(f)\right|\left\|\mathrm{h}_{l}(\cdot)\right\|_{L^{p}([0,1], w)}=0$. We skip the details because a similar result we have proved for the proof of Theorem 4.4.

In the case $p=1$ we have the following result. 
Theorem 5.3. For any $m=2,3, \ldots$ the system $\mathcal{H}_{0}(m)$ is a basis in the weighted norm space $L^{1}([0,1], w)$, if and only if $\frac{1}{w} \notin L^{\infty}([0,1])$ and there exists a point $y \in[0,1]$ such that $w$ satisfies the conditions $\mathcal{M}_{1}([0,1] \backslash\{y\})$ and $\mathcal{M}_{1}^{y}([0,1])$.

We will not give the details of the proof because it is similar to the proof of Theorem 5.2. The main theorem of this section is the following

Theorem 5.4. If the system $\mathcal{H}_{0}(m), m=2,3, \ldots$ is a basis in the weighted norm space $L^{p}([0,1], w), 1<p<\infty$ then $\mathcal{H}_{0}(m)$ is an unconditional basis in the same space.

Proof. By Theorem 5.2 we have that there exists $y \in[0,1]$ such that the weight function $w$ satisfies the conditions $\mathcal{M}_{p}([0,1] \backslash\{y\})$ and $\mathcal{M}_{p}^{y}([0,1])$. For any $f \in L^{p}([0,1], w)$ there exists a unique sequence $\left\{a_{l}(f)\right\}_{l=1}^{\infty}$ such that

$$
f=\sum_{l=1}^{\infty} a_{l}(f) \mathrm{h}_{l}
$$

The coefficients which correspond to the functions $h_{\Delta_{j}(y)}^{(\nu)}$ in the series (5.7) we denote by $b_{j \nu}$. We split formally the series (5.7) into two parts

$$
\sum_{l=1}^{\infty} a_{l}(f) \mathrm{h}_{l}=\sum_{j=0}^{\infty} \sum_{\nu=1}^{m-1} b_{j \nu}(f) h_{\Delta_{j}(y)}^{(\nu)}+\sum^{\prime} a_{l}(f) \mathrm{h}_{l},
$$

where by $\sum^{\prime}$ we have denoted the series obtained after excluding the terms which are present in the first series.

For any $k \in \mathbb{N}_{0}$ let $G_{k l} \subset \mathcal{M}, 1 \leq l \leq m-1$ be mutually disjoint intervals such that $\left|G_{k l}\right|=m^{-k-1}, 1 \leq l \leq m-1$ and $\Delta_{k}(y)=\Delta_{k+1}(y) \bigcup \bigcup_{l=1}^{m-1} G_{k l}$.

By Theorem 4.5 we easily obtain that the series $\sum^{\prime} a_{l}(f) \mathrm{h}_{l}$ converges unconditionally in $L^{p}\left(G_{k l}, w\right)$ for any $k \in \mathbb{N}$ and for all $1 \leq l \leq m-1$. Hence, if we check that the series $\sum^{\prime} a_{l}(f) \mathrm{h}_{l}$ converges in $L^{p}([0,1], w)$ we will have that it converges unconditionally in $L^{p}([0,1], w)$. Thus the proof of theorem will be finished if we prove that the first series on the right hand side of the equality (5.8) converges unconditionally in $L^{p}([0,1], w)$. Recall that we are using the notation introduced in (3.23). Let

$$
F(x)=\sum_{j=0}^{\infty} \sum_{\nu=1}^{m-1} b_{j \nu}(f) h_{\Delta_{j}(y)}^{(\nu)}(x)=d_{k}^{l} \quad \text { if } \quad x \in G_{k l} .
$$


for all $k \in \mathbb{N}_{0}$ and $1 \leq l \leq m-1$. By Lemma 5.4 we have that

$$
d_{k}^{l}=\frac{1}{\left|G_{k l}\right|} \int_{G_{k l}} f(t) d t, \quad \text { for } \quad x \in G_{k l} .
$$

The weight function $w$ satisfies the condition $\mathcal{M}_{p}([0,1] \backslash\{y\})$. Hence,

$$
\begin{gathered}
\int_{[0,1]}|F(x)|^{p} w(x) d x=\sum_{k=0}^{\infty} \sum_{l=1}^{m-1}\left|d_{k}^{l}\right|^{p} w\left(G_{k l}\right) \\
\leq \sum_{k=0}^{\infty} \sum_{l=1}^{m-1}\left|G_{k l}\right|^{-p} \int_{G_{k l}}|f(t)|^{p} w(t) d t\left(\int_{G_{k l}} w(t)^{-\frac{1}{p-1}} d t\right)^{p-1} w\left(G_{k l}\right) \\
\leq C_{p} \int_{[0,1]}|f(t)|^{p} w(t) d t .
\end{gathered}
$$

The system $\mathcal{H}_{0}(m)$ is a basis in the weighted norm space $L^{p}([0,1], w)$. Hence, the first series in the right hand side of the equality (5.8) converges in $L^{p}([0,1], w)$. Thus to finish the proof of Theorem 5.4 we have to prove that the series in (5.9) converge unconditionally in $L^{p}([0,1], w)$.

For any $j \in \mathbb{N}_{0}$ we have that

$$
\sum_{\nu=1}^{m-1} b_{j \nu}(f) h_{\Delta_{j}(y)}^{(\nu)}(x)=d_{j}^{l}=\frac{1}{\left|G_{j l}\right|} \int_{G_{j l}} f(t) d t
$$

for $x \in G_{j l}, 1 \leq l \leq m-1$ and

$$
\sum_{\nu=1}^{m-1} b_{j \nu}(f) h_{\Delta_{j}(y)}^{(\nu)}(x):=-c_{j}=-\sum_{l=1}^{m-1} d_{j}^{l}, \quad \text { for } \quad x \in \Delta_{j+1}(y) .
$$

Let $\left\{\gamma_{l}\right\}_{l=0}^{m-1}$ be a collection of numbers such that

$$
\sum_{l=0}^{m-1} \gamma_{l}^{2}=1 \quad \text { and } \quad \sum_{l=0}^{m-1} \gamma_{l}=0
$$

We put

$$
\xi_{j}(x)=\left|\Delta_{j+1}(y)\right|^{-\frac{1}{2}}\left[\gamma_{0} \chi_{\Delta_{j+1}(y)}(x)+\sum_{l=1}^{m-1} \gamma_{l} \chi_{G_{j l}}(x)\right]
$$


and

$$
\begin{gathered}
\alpha_{j}(f)=\int_{[0,1]} f(t)\left[\xi_{j}(t)-\left|\Delta_{j+1}(y)\right|^{-\frac{1}{2}} \gamma_{0}\right] d t \\
=-\frac{\gamma_{0}}{\sqrt{\left|\Delta_{j+1}(y)\right|}} \int_{[0,1] \backslash \Delta_{j+1}(y)} f(t) d t+\int_{[0,1] \backslash \Delta_{j+1}(y)} f(t) \xi_{j}(t) d t \\
=\left|\Delta_{j+1}(y)\right|^{\frac{1}{2}} \sum_{l=1}^{m-1}\left(\gamma_{l}-\gamma_{0}\right) d_{j}^{l}-\frac{\gamma_{0}}{\sqrt{\left|\Delta_{j+1}(y)\right|}} \int_{[0,1] \backslash \Delta_{j}(y)} f(t) d t . \\
=\left|\Delta_{j+1}(y)\right|^{\frac{1}{2}}\left(\sum_{l=1}^{m-1}\left(\gamma_{l}-\gamma_{0}\right) d_{j}^{l}-\gamma_{0} \sum_{s=0}^{j-1} m^{j-s-1} c_{s}\right) .
\end{gathered}
$$

Lemma 5.6. For any $\varepsilon=\left\{\epsilon_{j}\right\}_{j=0}^{\infty}$ let

$$
F_{\varepsilon}^{*}(x)=\sum_{j=0}^{\infty} \epsilon_{j} \alpha_{j} \xi_{j}(x) .
$$

Then for all $k \in \mathbb{N}$ and $x \in \Delta_{k}(y) \backslash \Delta_{k+1}(y)$

$$
\left|F_{\varepsilon}^{*}(x)\right| \leq 2 \sum_{s=0}^{k} \sum_{l=1}^{m-1}\left|d_{s}^{l}\right|+\frac{1}{m-1} \sum_{s=0}^{k-1} m^{k-s}\left|c_{s}\right| .
$$

Proof. By (5.11) and (5.12) we obtain that for $x \in G_{k \nu}, 1 \leq \nu \leq m-1$

$$
\begin{gathered}
\left|F_{\varepsilon}^{*}(x)\right| \leq \sum_{j=0}^{k}\left|\alpha_{j} \xi_{j}(x)\right|=\left|\gamma_{0}\right| \sum_{s=0}^{k-1} \sum_{l=1}^{m-1}\left|\gamma_{l}-\gamma_{0}\right|\left|d_{s}^{l}\right|+\left|\gamma_{\nu}\right| \sum_{l=1}^{m-1}\left|\gamma_{l}-\gamma_{0}\right|\left|d_{k}^{l}\right| \\
+\sum_{j=1}^{k-1} \sum_{s=0}^{j-1} m^{j-s-1}\left|c_{s}\right| \leq 2 \sum_{s=0}^{k} \sum_{l=1}^{m-1}\left|d_{s}^{l}\right|+\sum_{j=1}^{k-1} \sum_{s=0}^{j-1} m^{j-s-1}\left|c_{s}\right| \\
\leq 2 \sum_{s=0}^{k} \sum_{l=1}^{m-1}\left|d_{s}^{l}\right|+\frac{1}{m-1} \sum_{s=0}^{k-1} m^{k-s}\left|c_{s}\right| .
\end{gathered}
$$

Lemma 5.7. For any $f \in L^{p}([0,1], w), 1<p<\infty$ and any $\varepsilon=\left\{\epsilon_{j}\right\}_{j=0}^{\infty}$ the function $F_{\varepsilon}^{*} \in L^{p}([0,1], w)$ and

$$
\left\|F_{\varepsilon}^{*}\right\|_{L^{p}([0,1], w)} \leq C_{p}^{\prime}\|f\|_{L^{p}([0,1], w)},
$$

where $C_{p}^{\prime}>0$ is independent of $f$ and $\varepsilon$. 
Proof. By Lemma 5.6 we have that

$$
\begin{gathered}
\int_{\Delta_{k}(y) \backslash \Delta_{k+1}(y)}\left|F^{*}(x)\right|^{p} w(t) d t \leq 4^{p}\left(\sum_{s=0}^{k} \sum_{l=1}^{m-1}\left|d_{s}^{l}\right|\right)^{p} w\left(\Delta_{k}(y)\right) \\
+\frac{2^{p}}{(m-1)^{p}}\left(\left[w\left(\Delta_{k}(y)\right)\right]^{\frac{1}{p}} \sum_{s=0}^{k-1} m^{k-s}\left|c_{s}\right|\right)^{p} .
\end{gathered}
$$

Afterwards write

$$
\begin{gathered}
\left|\Delta_{s+1}(y)\right| \sum_{l=1}^{m-1}\left|d_{s}^{l}\right|\left[w\left(\Delta_{k}(y)\right)\right]^{\frac{1}{p}} \\
\leq\left(\int_{\Delta_{s}(y) \backslash \Delta_{s+1}(y)}|f(t)|^{p} w(t) d t\right)^{\frac{1}{p}}\left(\int_{\Delta_{s}(y) \backslash \Delta_{s+1}(y)} w(t)^{-\frac{1}{p-1}} d t\right)^{\frac{1}{p^{\prime}}}\left[w\left(\Delta_{k}(y)\right)\right]^{\frac{1}{p}} . \\
\leq C_{p}^{\frac{1}{p}}\left(\int_{\Delta_{s}(y) \backslash \Delta_{s+1}(y)}|f(t)|^{p} w(t) d t\right)^{\frac{1}{p}}\left|\Delta_{k}(y)\right| .
\end{gathered}
$$

Hence, we obtain that

$$
\sum_{s=0}^{k} \sum_{l=1}^{m-1}\left|d_{s}^{l}\right|\left[w\left(\Delta_{k}(y)\right)\right]^{\frac{1}{p}} \leq C_{p}^{\frac{1}{p}}\left(\int_{\Delta_{s}(y) \backslash \Delta_{s+1}(y)}|f(t)|^{p} w(t) d t\right)^{\frac{1}{p}} \frac{1}{m^{k-s}}
$$

Now we apply the following lemma which is a consequence of Theorem 274 from [11].

Lemma 5.8. Let $u=\left\{u_{j}\right\}_{j=0}^{\infty}$ and $v=\left\{v_{j}\right\}_{j=0}^{\infty}$ be numerical sequences such that $u \in l^{1}$ and $v \in l^{p}, p>1$. Then the Cauchy product $w=\left\{w_{n}\right\}_{n=0}^{\infty}$, $w_{n}=\sum_{j=0}^{n} u_{n-j} v_{j}$ of the sequences $u$ and $v$ belongs to $l^{p}$. Moreover $\|w\|_{l^{p}} \leq\|u\|_{l^{1}}\|v\|_{l^{p}}$.

Which gives us the convergence of the series

$$
\sum_{k=1}^{\infty}\left(\sum_{s=0}^{k} \sum_{l=1}^{m-1}\left|d_{s}^{l}\right|\right)^{p} w\left(\Delta_{k}(y)\right) \leq 2^{p} C_{p} \int_{[0,1]}|f(t)|^{p} w(t) d t
$$

To finish the proof of Lemma 5.7 we have to show that

$$
\sum_{k=1}^{\infty}\left(\left[w\left(\Delta_{k}(y)\right)\right]^{\frac{1}{p}} \sum_{s=0}^{k-1} m^{k-s}\left|c_{s}\right|\right)^{p}<+\infty
$$


We have that

$$
\begin{gathered}
\left(\sum_{s=0}^{k-1} m^{-s-1}\left|c_{s}\right|\right)^{p} \\
\leq\left(\sum_{s=0}^{k-1} \sum_{l=1}^{m-1}\left(\int_{G_{s l}}|f(t)|^{p} w(t) d t\right)^{\frac{1}{p}}\left(\int_{G_{s l}} w(t)^{-\frac{1}{p-1}} d t\right)^{\frac{1}{p^{\prime}}}\right)^{p} \\
\leq\left(\sum_{s=0}^{k-1}\left(\int_{\Delta_{s}(y) \backslash \Delta_{s+1}(y)}|f(t)|^{p} w(t) d t\right)^{\frac{1}{p}}\right. \\
\left.\times\left(\int_{\Delta_{s}(y) \backslash \Delta_{s+1}(y)} w(t)^{-\frac{1}{p-1}} d t\right)^{\frac{1}{p^{\prime}}}\right)^{p} .
\end{gathered}
$$

Recall that $w$ satisfies $\mathcal{M}_{p}^{y}([0,1])$. By Lemma 3.6 we obtain that

$$
\begin{gathered}
m^{k}\left(\int_{\Delta_{s}(y) \backslash \Delta_{s+1}(y)} w(t)^{-\frac{1}{p-1}} d t\right)^{\frac{1}{p^{\prime}}} w\left(G_{k l}\right)^{\frac{1}{p}} \\
\leq C_{p}^{\frac{1}{p}}\left(\int_{\Delta_{s}(y) \backslash \Delta_{s+1}(y)} w(t)^{-\frac{1}{p-1}} d t\right)^{\frac{1}{p^{\prime}}}\left[\int_{[0,1] \backslash \Delta_{k}(y)} \omega^{\left.-\frac{1}{p-1}(t) d t\right]^{-\frac{1}{p^{\prime}}}}\right. \\
\leq C_{p}^{\frac{1}{p}} q_{p}^{-\frac{k-s}{p}} .
\end{gathered}
$$

If we write

$$
\begin{gathered}
\sum_{s=0}^{k-1} m^{k-s-1}\left|c_{s}\right| w\left(G_{k l}\right)^{\frac{1}{p}} \\
\leq C_{p}^{\frac{1}{p}} \sum_{s=0}^{k-1}\left(\int_{\Delta_{s}(y) \backslash \Delta_{s+1}(y)}|f(t)|^{p} w(t) d t\right)^{\frac{1}{p}} q_{p}^{-\frac{k-s}{p}}
\end{gathered}
$$

and put $v_{j}=\left(\int_{\Delta_{j}(y) \backslash \Delta_{j+1}(y)}|f(t)|^{p} w(t) d t\right)^{\frac{1}{p}}, u_{j}=q_{p}^{-\frac{j}{p}}$ then by Lemma 5.8 we will obtain

$$
\sum_{k=1}^{\infty}\left(\sum_{s=0}^{k-1} m^{k-s-1}\left|c_{s}\right|\right)^{p} w\left(G_{k l}\right) \leq C_{p} B_{p} \int_{[0,1]}|f(t)|^{p} w(t) d t
$$


Lemma 5.7 yields the convergence of the series

$$
\sum_{j=0}^{\infty} \epsilon_{j}^{(\nu)} b_{j \nu}(f) h_{\Delta_{j}(y)}^{(\nu)}
$$

for any $1 \leq \nu \leq m-1$ and $\forall \varepsilon^{(\nu)}=\left\{\epsilon_{j}^{(\nu)}\right\}_{j=0}^{\infty}$, where $\epsilon_{j}^{(\nu)}= \pm 1$. Moreover, we obtain that for some $B_{p}>0$

$$
\left\|\sum_{j=0}^{\infty} \sum_{\nu=1}^{m-1} \epsilon_{j}^{(\nu)} b_{j \nu}(f) h_{\Delta_{j}(y)}^{(\nu)}\right\|_{L^{p}([0,1], w)} \leq B_{p}\|f\|_{L^{p}([0,1], w)} .
$$

\section{Higher rank Haar wavelets in $L^{p}(\mathbb{R}, \omega)$}

Let $\omega \geq 0$ be a locally integrable function defined on $\mathbb{R}$. In this section we study the phenomenon described in the introduction with respect to the higher rank Haar wavelet systems $H(m), m=2,3, \ldots$ Let $\chi^{-}(x)=\chi_{\mathbb{R}^{-}}(x)$ and $\chi^{+}(x)=\chi_{\mathbb{R}^{+}}(x)$. The following result is the first step in that direction.

Lemma 6.1. For any $m=2,3, \ldots$ let $H(m)$ be the wavelet system defined by (3.21) and (3.22). Let $U_{m}$ be the linear subspace of locally integrable functions $\xi$ on $\mathbb{R}$ such that

$$
\int_{\mathbb{R}} \xi(t) h_{k, j, m}^{(\nu)}(x)(t) d t=0 \quad \forall j, k \in \mathbb{Z}, 1 \leq \nu \leq m-1 .
$$

Then $\operatorname{dim} U_{m}=2$ and $\chi^{-}, \chi^{+}$as vectors constitute a basis in $U_{m}$.

Proof. It is clear that if we prove that a locally integrable function $\xi$ such that $\xi(x)=0$ if $x \in \mathbb{R}^{-}$and holds (6.1) if and only if $\xi=c \chi^{+}$for some $c \in \mathbb{R}$ then the proof will be finished. By Corollary 4.3 we have that the system $\mathcal{H}(m), m=2,3, \ldots$ is total with respect to $L^{1}[0,1]$. Hence, by definition of the system $\mathcal{H}(m)$ and by (6.1) it follows that

$$
\int_{[0.1]} \xi(t) \mathrm{h}_{l}(t) d t=0 \quad \text { for all } \quad l \in \mathbb{N} .
$$

Which yields that $\xi(x)=c \mathrm{~h}_{0}(x)$ for $x \in[0,1]$. We finish the proof by induction. Suppose that for some $N \in \mathbb{N}$ it is true that if $\xi$ is a locally 
integrable function such that $\xi(x)=0$ if $x \in \mathbb{R}^{-}$and (6.1) is true then $\xi(x)=c_{0}$ if $x \in\left[0, m^{N}\right]$, where $c_{0} \in \mathbb{R}$. If $\xi$ is a function which satisfies to all mentioned conditions then by definition of the system $H(m)$ it follows that the functions $\xi_{\nu}(x)=\xi\left(x-\nu m^{N}\right), 1 \leq \nu \leq m-1$. Thus by our supposition it follows that $\xi_{\nu}(x)=c_{\nu}$ if $x \in\left[0, m^{N}\right]$, where $c_{\nu} \in \mathbb{R}$. Hence, $\xi(x)=c_{\nu}$ if $x \in\left[\nu m^{N},(\nu+1) m^{N}\right], 0 \leq \nu \leq m-1$. Afterwards we observe that the functions $h^{(\nu)}\left(m^{N+1} x\right), 1 \leq \nu \leq m-1$ belong to the system $H(m)$, which yields

$$
\int_{0}^{m^{N+1}} \xi(x) h^{(\nu)}\left(m^{N+1} x\right) d x=0 \quad \text { for all } \quad 1 \leq \nu \leq m-1 .
$$

After a change of the variable we have that

$$
\int_{0}^{1} \xi\left(m^{-N-1} t\right) h^{(\nu)}(t) d t=0 \quad \text { for all } \quad 1 \leq \nu \leq m-1 .
$$

By definition of the functions $h^{(\nu)}, 1 \leq \nu \leq m-1$ we obtain that $\xi\left(m^{-N-1} x\right)=$ $c$ if $x \in[0,1]$.

It is convenient to continue our study considering the systems $H^{+}(m)$, $H^{-}(m)$ respectively in the spaces $L^{p}\left(\mathbb{R}^{+}, \omega\right)$ and $L^{p}\left(\mathbb{R}^{-}, \omega\right)$. It is easy to see some sort of symmetry between those systems. Thus it would be sufficient to study the system $H^{+}(m)$ in the space $L^{p}\left(\mathbb{R}^{+}\right)$. In fact we have proved the analogue of the above lemma for the system $\mathrm{H}^{+}(m)$ which is formulated as follows.

Lemma 6.2. Let $U_{m}^{+}$be the linear subspace of locally integrable functions $\xi$ on $\mathbb{R}^{+}$such that

$$
\int_{\mathbb{R}^{+}} \xi(t) h_{k, j, m}^{(\nu)}(x)(t) d t=0 \quad \forall k \in \mathbb{Z}, \forall j \in \mathbb{Z}^{+}, 1 \leq \nu \leq m-1 .
$$

Then $\operatorname{dim} U_{m}^{+}=1$ and $\chi^{+} \in U_{m}^{+}$.

We need the analogues of Lemmas 5.1, 5.2 for this case.

Lemma 6.3. The system $H^{+}(m)$ is complete in $L^{p}\left(\mathbb{R}^{+}, \omega\right), 1 \leq p<\infty$ if and only if

$$
\frac{\chi^{+}}{\omega} \notin L^{\frac{1}{p-1}}\left(\mathbb{R}^{+}\right)
$$


Proof. Suppose that $H^{+}(m)$ is complete in $L^{p}\left(\mathbb{R}^{+}, \omega\right)$. If $g=\frac{\chi^{+}}{\omega} \in L^{\frac{1}{p-1}}\left(\mathbb{R}^{+}\right)$ then $g \in L^{p^{\prime}}\left(\mathbb{R}^{+}, \omega\right)$, where $\frac{1}{p}+\frac{1}{p^{\prime}}=1$. Thus

$$
\int_{\mathbb{R}^{+}} g(t) h_{k, j, m}^{(\nu)}(x)(t) \omega(t) d t=0 \quad \forall k \in \mathbb{Z}, \forall j \in \mathbb{Z}^{+}, 1 \leq \nu \leq m-1 .
$$

Which yields that $H^{+}(m)$ is not complete in $L^{p}\left(\mathbb{R}^{+}, \omega\right)$. Which is a contradiction.

Suppose that $\frac{\chi^{+}}{\omega} \notin L^{\frac{1}{p-1}}\left(\mathbb{R}^{+}\right)$. If $H^{+}(m)$ is not complete in $L^{p}\left(\mathbb{R}^{+}, \omega\right)$ then there exists $g \in L^{p^{\prime}}\left(\mathbb{R}^{+}, \omega\right)$ such that (6.4) holds. By Lemma 6.3 it follows that $g(t) \omega(t)=c \chi^{+}(t)$ a.e. on $\mathbb{R}^{+}$, where $c \in \mathbb{R}$. We came to a contradiction which finishes the proof.

From Lemma 6.3 follows

Lemma 6.4. The system $H^{+}(m)$ is complete in $L^{p}\left(\mathbb{R}^{+}, \omega\right), 1 \leq p<\infty$ if and only if there exists at least one point $y \in[0,+\infty]$ such that

$$
\frac{1}{w} \notin L^{\frac{1}{p-1}}\left(\Delta_{j}(y)\right) \quad \text { for all } \quad j \in \mathbb{N} \text {. }
$$

We also have

Lemma 6.5. The system $H^{+}(m)$ is minimal in $L^{p}\left(\mathbb{R}^{+}, \omega\right), 1 \leq p<\infty$ if and only if

For any $h_{k, j, m}^{(\nu)}(x) \in H^{+}(m)$ there exists a coefficient $a_{k, j, m}^{(\nu)}$ such that

$$
g_{k, j, m}^{(\nu)}=\frac{a_{k, j, m}^{(\nu)} \chi^{+}+h_{k, j, m}^{(\nu)}}{\omega} \in L^{\frac{1}{p-1}}\left(\mathbb{R}^{+}\right) .
$$

Proof. Suppose that the system $H^{+}(m)$ is minimal in $L^{p}\left(\mathbb{R}^{+}, \omega\right)$. Then there exists a system $\left\{g_{k, j, m}^{(\nu)}: k \in \mathbb{Z}, j \in \mathbb{Z}^{+}, 1 \leq \nu \leq m-1\right\}$ biorthogonal to $H^{+}(m)$. Hence, if for some $\nu_{0}, 1 \leq \nu_{0} \leq m-1$ we fix any $l \in \mathbb{Z}$ and any $\mu \in \mathbb{Z}^{+}$then for all $k \in \mathbb{Z}, j \in \mathbb{Z}^{+}$and $1 \leq \nu \leq m-1$

$$
\int_{\mathbb{R}^{+}}\left[g_{l, \mu, m}^{\left(\nu_{0}\right)}(x) \omega(x)-h_{l, \mu, m}^{\left(\nu_{0}\right)}(x)\right] h_{k, j, m}^{(\nu)}(x) d x=0 .
$$


By Lemma 6.2 we obtain that

$$
g_{l, \mu, m}^{\left(\nu_{0}\right)}=\frac{a_{l, \mu, m}^{\left(\nu_{0}\right)} \chi^{+}+h_{l, \mu, m}^{\left(\nu_{0}\right)}}{\omega} \in L^{\frac{1}{p-1}}\left(\mathbb{R}^{+}\right) .
$$

The proof of sufficiency is direct. We easily check that the system $\left\{g_{k, j, m}^{(\nu)}: k \in \mathbb{Z}, j \in \mathbb{Z}^{+}, 1 \leq \nu \leq m-1\right\}$ is biorthogonal to $H^{+}(m)$.

From Lemma 6.5 easily follows

Lemma 6.6. The system $H^{+}(m)$ is minimal in $L^{p}\left(\mathbb{R}^{+}, \omega\right), 1 \leq p<\infty$ if and only if there exists at most one point $y \in[0,+\infty]$ such that (6.5) holds.

By Lemmas 6.3 and 6.5 we obtain immediately

Lemma 6.7. The system $H^{+}(m)$ is complete and minimal in $L^{p}\left(\mathbb{R}^{+}, \omega\right)$, $1 \leq p<\infty$ if and only if conditions (6.3) and (M) hold.

Lemmas 6.4 and 6.6 yield

Lemma 6.8. The system $H^{+}(m)$ is complete and minimal in $L^{p}\left(\mathbb{R}^{+}, \omega\right)$, $1 \leq p<\infty$ if and only if there exists a unique point $y \in[0,+\infty]$ such that the condition (6.5) holds.

If we analyze the proofs of results which brought us the last lemma then it is not hard to see that the following result also holds.

Lemma 6.9. The system $H^{-}(m)$ is complete minimal in $L^{p}\left(\mathbb{R}^{-}, \omega\right), 1 \leq$ $p<\infty$ if and only if there exists a unique point $y \in[-\infty, 0]$ such that the condition (6.5) holds.

Lemma 6.8 and Lemma 6.9 easily yield

Lemma 6.10. The system $H(m)$ is complete and minimal in $L^{p}(\mathbb{R}, \omega), 1 \leq$ $p<\infty$ if and only if there exists a unique point $y^{+} \in[0,+\infty]$ and a unique point $y^{-} \in[-\infty, 0]$ such that the condition (6.5) holds for both of those points.

The following lemma will be used in the proof of the main result of the present section. 
Lemma 6.11. Let $\left\{\phi_{j}\right\}_{j=1}^{\mu},\left\{\psi_{j}\right\}_{j=1}^{\mu}$ be some measurable functions defined on a measurable set $E$ and let

$$
K(x, t)=\sum_{j=1}^{\mu} \phi_{j}(x) \psi_{j}(t) \quad(x, t) \in E \times E .
$$

Furthermore, for a given real valued orthogonal matrix

$$
A=\left(a_{i j}\right)_{\substack{1 \leq i \leq \mu \\ 1 \leq j \leq \mu}}
$$

let $f_{k}(x)=\sum_{i=1}^{\mu} a_{i k} \phi_{i}(x), g_{k}(x)=\sum_{i=1}^{\mu} a_{i k} \psi_{i}(t)$. If we consider a new kernel $\Phi(x, t)=\sum_{k=1}^{\mu} f_{k}(x) g_{k}(t)$ then

$$
K(x, t)=\Phi(x, t) \quad \text { for } \quad(x, t) \in E \times E .
$$

Proof. We have

$$
\begin{aligned}
\Phi(x, t) & =\sum_{k=1}^{\mu} \sum_{i=1}^{\mu} a_{i k} \phi_{i}(x) \sum_{\nu=1}^{\mu} a_{\nu k} \psi_{\nu}(t) \\
& =\sum_{i=1}^{\mu} \sum_{\nu=1}^{\mu} \phi_{i}(x) \psi_{\nu}(t) \sum_{k=1}^{\mu} a_{\nu k} a_{i k} \\
& =\sum_{\nu=1}^{\mu} \phi_{\nu}(x) \psi_{\nu}(t)=K(x, t) .
\end{aligned}
$$

We are going to apply Lemma 6.11 in the proof of the next theorem. As $\left\{\phi_{j}\right\}_{j=1}^{\mu}$ and $\left\{f_{k}\right\}_{k=1}^{\mu}$ we will take two orthonormal bases in $V(m)$ considered in Section 3.2. Concretely we will consider the following orthonormal bases of $V(m):\left\{h^{(\nu)}(x): 0 \leq \nu \leq m-1\right\}$ and $\left\{\varphi_{1, j, m}(x): 0 \leq j \leq m-1\right\}$.

Theorem 6.1. For any $m=2,3, \ldots$ the system $H^{+}(m)$ is an unconditional basis in the weighted norm space $L^{p}\left(\mathbb{R}^{+}, \omega\right), 1<p<\infty$ if and only if there exists a point $y \in[0,+\infty]$ such that:

If $y \neq+\infty$ then $\omega$ satisfies the condition $\mathcal{M}_{p}\left(\mathbb{R}^{+} \backslash\{y\}\right)$ and the condition $\mathcal{M}_{p}^{y}\left(\mathbb{R}^{+}\right)$

If $y=+\infty$ then $\omega$ satisfies the condition $\mathcal{M}_{p}\left(\mathbb{R}^{+}\right)$. 
Proof. Suppose that $H^{+}(m)$ is an unconditional basis in the weighted norm space $L^{p}\left(\mathbb{R}^{+}, \omega\right), 1<p<\infty$. Then $H^{+}(m)$ is complete minimal in $L^{p}\left(\mathbb{R}^{+}, \omega\right)$ and there exists a unique point $y \in[0,+\infty]$ such that the condition (6.5) holds. First consider the case $y=+\infty$. In this case the uniqueness of the point $y$ means that for any $h_{k, j, m}^{(\nu)}(x) \in H^{+}(m)$

$$
g_{k, j, m}^{(\nu)}=\frac{h_{k, j, m}^{(\nu)}}{\omega} \in L^{\frac{1}{p-1}}\left(\mathbb{R}^{+}\right) .
$$

The proof of the necessity can be easily completed following the scheme of the proof of Theorem 4.4. If $y \in[0,+\infty)$ then by Lemma 6.5 the system biorthogonal to $H^{+}(m)$ is defined by the following equations:

$$
g_{k, j, m}^{(\nu)}(t)=\frac{h_{k, j, m}^{(\nu)}(t)-h_{k, j, m}^{(\nu)}(y) \chi^{+}(t)}{\omega(t)}
$$

for all $k \in \mathbb{Z}, j \in \mathbb{Z}^{+}$and $1 \leq \nu \leq m-1$.

Let $\Delta=\Delta_{l+1}(y)$ and let $\Delta_{l}(y)=\Delta_{l}(0)+j_{y} m^{-l}$, where $j_{y} \in \mathbb{N}_{0}$. For $f \in L^{p}\left(\mathbb{R}^{+}, w\right)$, consider the sum

$$
\begin{aligned}
& \sum_{\nu=1}^{m-1} c_{l, j_{y}, m}^{(\nu)}(f) h_{l, j_{y}, m}^{(\nu)}(x) \\
& =\sum_{\nu=0}^{m-1} \int_{\mathbb{R}^{+}} f(t) g_{k, j_{y}, m}^{(\nu)}(t) \omega(t) d t h_{l, j_{y}, m}^{(\nu)}(x)-c_{l, j_{y}, m}^{(0)}(f) h_{l, j_{y}, m}^{(0)}(x) \\
& =\int_{\mathbb{R}^{+}} f(t) \sum_{\nu=0}^{m-1} g_{k, j_{y}, m}^{(\nu)}(t) h_{l, j_{y}, m}^{(\nu)}(x) \omega(t) d t-c_{l, j_{y}, m}^{(0)}(f) h_{l, j_{y}, m}^{(0)}(x),
\end{aligned}
$$

where $h_{l, j_{y}, m}^{(0)}(x)=\chi_{\Delta_{l}(y)}(x)$ and

$$
g_{k, j, m}^{(0)}(t)=\frac{h_{k, j, m}^{(0)}(t)-h_{k, j, m}^{(0)}(y) \chi^{+}(t)}{\omega(t)} .
$$

By Lemma 6.11 follows that

$$
\begin{aligned}
& \int_{\mathbb{R}^{+}} f(t) \sum_{\nu=0}^{m-1} g_{k, j_{y}, m}^{(\nu)}(t) h_{l, j_{y}, m}^{(\nu)}(x) \omega(t) d t \\
& =\int_{\mathbb{R}^{+}} f(t) \sum_{j=0}^{m-1} \varphi_{l+1, j_{y}+j, m}(x) \psi_{l, j, m}(t) \omega(t) d t
\end{aligned}
$$


where

$$
\psi_{l, j, m}(t)=\frac{\varphi_{l+1, j_{y}+j, m}(t)-\varphi_{l+1, j_{y}+j, m}(y) \chi^{+}(t)}{\omega(t)} .
$$

Thus we obtain that if $x \in \Delta_{l+1}(y)$ then

$$
\sum_{\nu=1}^{m-1} c_{l, j_{y}, m}^{(\nu)}(f) h_{l, j_{y}, m}^{(\nu)}(x)=-m^{l+1} \int_{\mathbb{R}^{+} \backslash \Delta_{l+1}(y)} f(t) d t-m^{l} \int_{\mathbb{R}^{+} \backslash \Delta_{l}(y)} f(t) d t .
$$

If $f(t) \geq 0$ for $t \in \mathbb{R}^{+}$then it follows that for $x \in \Delta_{l+1}(y)$

$$
\left|\sum_{\nu=1}^{m-1} c_{l, j_{y}, m}^{(\nu)}(f) h_{l, j_{y}, m}^{(\nu)}(x)\right| \geq\left|\Delta_{l+1}(y)\right|^{-1}\left|\int_{\mathbb{R}^{+} \backslash \Delta_{l+1}(y)} f(t) d t\right| .
$$

Afterwards in the same way as in the proof of Theorem 5.2 we obtain that for some $B_{p}>0$ and for all $l \in \mathbb{Z}$

$$
\left|\Delta_{l}(y)\right|^{-p} \omega\left(\Delta_{l}(y)\right)\left(\int_{\mathbb{R}^{+} \backslash \Delta_{l}(y)} \omega^{-\frac{1}{p-1}} d t\right)^{p-1} \leq B_{p}^{p} .
$$

Let $E_{l j} \in \mathcal{M}, 1 \leq j \leq m-1$ be mutually disjoint intervals such that $\left|E_{l j}\right|=$ $m^{-1}\left|\Delta_{l}(y)\right|, 1 \leq l \leq m-1$ and $\Delta_{l}(y)=\Delta_{l+1}(y) \cup \bigcup_{j=1}^{m-1} E_{l j}$. By (6.9) we obtain that if $f(t)=0$ when $x \in \mathbb{R}^{+} \backslash \Delta_{l}(y)$ then

$$
\sum_{\nu=1}^{m-1} c_{l, j_{y}, m}^{(\nu)}(f) h_{l, j_{y}, m}^{(\nu)}(x)=\left|E_{l j}\right|^{-1} \int_{E_{l j}} f(t) d t \quad \text { if } \quad x \in E_{l j}(1 \leq j \leq m-1) .
$$

Which yields

$$
\left|E_{l j}\right|^{-p} \omega\left(E_{l j}\right)\left(\int_{E_{l j}} \omega^{-\frac{1}{p-1}} d t\right)^{p-1} \leq B_{p}^{p} \quad \text { for any } l \in \mathbb{Z} \quad \text { and } 1 \leq j \leq m-1 .
$$

Let $\Delta \in \mathcal{M},|\Delta|=m^{-l-1}$ be such that $\Delta \bigcap \Delta_{l}(y)=\emptyset$. We consider the interval $\Delta^{*} \in \mathcal{M},\left|\Delta^{*}\right|=m^{-l}$ be such that $\Delta \subset \Delta^{*}$. Let $=\Delta_{l}(0)+k^{*} m^{-l}$, where $k^{*} \in \mathbb{N}_{0}$.

For $f \in L^{p}\left(\mathbb{R}^{+}, w\right)$ consider the sum

$$
\sum_{\nu=1}^{m-1} c_{l, k^{*}, m}^{(\nu)}(f) h_{l, k^{*}, m}^{(\nu)}(x)
$$


Using the same idea as above we show that if $x \in \Delta$ then

$$
\sum_{\nu=1}^{m-1} c_{l, k^{*}, m}^{(\nu)}(f) h_{l, k^{*}, m}^{(\nu)}(x)=|\Delta|^{-1} \int_{\Delta} f(t) d t-\left|\Delta^{*}\right|^{-1} \int_{\Delta^{*}} f(t) d t .
$$

Thus if $f(t)=0$ for $t \in \mathbb{R}^{+} \backslash \Delta$ it follows that

$$
\sum_{\nu=1}^{m-1} c_{l, k^{*}, m}^{(\nu)}(f) h_{l, k^{*}, m}^{(\nu)}(x)=\frac{m-1}{m}|\Delta|^{-1} \int_{\Delta} f(t) d t
$$

and the proof of the necessity is completed easily.

The proof of the sufficiency will be given following the same idea as in proof of Theorem 4.2. By Lemma 6.8 we have that the system $H^{+}(m)$ is complete and minimal in $L^{p}\left(\mathbb{R}^{+}, \omega\right)$. Let $G^{+}(m)=\left\{g_{k, j, m}^{(\nu)}: k \in \mathbb{Z}, j \in\right.$ $\left.\mathbb{Z}^{+}, 1 \leq \nu \leq m-1\right\}$ be the conjugate system of the basis $H^{+}(m)$. Suppose that $\omega$ satisfies the condition $\mathcal{M}_{p}\left(\mathbb{R}^{+}\right)$. Then the system $G^{+}(m)$ is defined by the equations (6.7). Let $f \in L^{p}\left(\mathbb{R}^{+}, \omega\right)$ and let $\Omega \subset \mathbb{Z} \times \mathbb{Z}^{+}$be a finite set. Moreover, let $N \in \mathbb{N}$ be such that $h_{k, j, m}^{(\nu)}(x)=0$ if $x \in\left[m^{N},+\infty\right)$ for all $(k, j) \in \Omega$. For any $1 \leq \nu \leq m-1$ consider the sum

$$
S_{\Omega}^{(\nu)}(f, x)=\sum_{(k, j) \in \Omega} c_{k j}^{(\nu)}(f) h_{k, j, m}^{(\nu)}(x),
$$

where

$$
c_{k j}^{(\nu)}(f)=\int_{\mathbb{R}^{+}} f(t) h_{k, j, m}^{(\nu)}(t) d t .
$$

Applying Lemma 3.7 as in the proof of Theorem 4.2 we obtain that for some

$$
\left\|S_{\Omega}^{(\nu)}(f, \cdot)\right\|_{L^{p}\left(\mathbb{R}^{+}, \omega\right)} \leq B_{p}\|f\|_{L^{p}\left(\mathbb{R}^{+}, \omega\right)}
$$

where $B_{p}>0$ is independent of $f$ and $\Omega$.

If $y \in[0,+\infty)$ then we take $N \in \mathbb{N}$ so that $h_{k, j, m}^{(\nu)}(x)=0$ if $x \in\left[m^{N},+\infty\right)$ for all $(k, j) \in \Omega$ and $y \in\left[0, m^{N}\right]$. By Lemma 6.5 the system $G^{+}(m)$ is defined by the equations (6.8). In this case the coefficients of the sum (6.11) are defined as follows:

$$
c_{k j}^{(\nu)}(f)=\int_{\mathbb{R}^{+}} f(t)\left[h_{k, j, m}^{(\nu)}(t)-h_{k, j, m}^{(\nu)}(y)\right] d t .
$$


We write $f(t)=f_{1}(t)+f_{2}(t)$, where $f_{1}(t)=f(t) \chi_{\left[0, m^{N}\right]}(t)$. By Lemma 3.8 and Lemma 3.9 in the same way as above we obtain that

$$
\left\|S_{\Omega}^{(\nu)}\left(f_{1}, \cdot\right)\right\|_{L^{p}\left(\mathbb{R}^{+}, \omega\right)} \leq B_{p}\left\|f_{1}\right\|_{L^{p}\left(\mathbb{R}^{+}, \omega\right)},
$$

where $B_{p}>0$ is independent of $f_{1}$ and $\Omega$. The proof will be complete if we show that

$$
\left\|S_{\Omega}^{(\nu)}\left(f_{2}, \cdot\right)\right\|_{L^{p}\left(\mathbb{R}^{+}, \omega\right)} \leq B_{p}^{*}\left\|f_{2}\right\|_{L^{p}\left(\mathbb{R}^{+}, \omega\right)},
$$

where $B_{p}^{*}>0$ is independent of $f_{2}$ and $\Omega$. We have that

$$
\begin{gathered}
S_{\Omega}^{(\nu)}\left(f_{2}, x\right)=\sum_{(k, j) \in \Omega} c_{k j}^{(\nu)}\left(f_{2}\right) h_{k, j, m}^{(\nu)}(x) \\
=-\int_{\left[m^{N},+\infty\right)} f(t) d t \sum_{(k, j) \in \Omega} h_{k, j, m}^{(\nu)}(y) h_{k, j, m}^{(\nu)}(x) .
\end{gathered}
$$

Let $f_{N}^{*}(t)=m^{-N} \chi_{\left[0, m^{N}\right]}(t)$ then

$$
\begin{aligned}
\sum_{(k, j) \in \Omega} h_{k, j, m}^{(\nu)}(y) h_{k, j, m}^{(\nu)}(x) & =\sum_{(k, j) \in \Omega} \int_{\mathbb{R}^{+}} f_{N}^{*}(t)\left[h_{k, j, m}^{(\nu)}(t)-h_{k, j, m}^{(\nu)}(y)\right] d t h_{k, j, m}^{(\nu)}(x) \\
& =S_{\Omega}^{(\nu)}\left(f_{N}^{*}, x\right) .
\end{aligned}
$$

Thus we have that

$$
\begin{aligned}
& \left\|S_{\Omega}^{(\nu)}\left(f_{2}, \cdot\right)\right\|_{L^{p}\left(\mathbb{R}^{+}, \omega\right)}=\left|\int_{\left[m^{N},+\infty\right)} f(t) d t\right|\left\|S_{\Omega}^{(\nu)}\left(f_{N}^{*}, \cdot\right)\right\|_{L^{p}\left(\mathbb{R}^{+}, \omega\right)} \\
& \leq\left\|f_{2}\right\|_{L^{p}\left(\mathbb{R}^{+}, \omega\right)}\left[\int_{\left[m^{N},+\infty\right)} \omega(t)^{-\frac{1}{p-1}} d t\right]^{\frac{1}{p^{\prime}}} B_{p}\left\|f_{N}^{*}\right\|_{L^{p}\left(\mathbb{R}^{+}, \omega\right)} \\
& =B_{p} m^{-N}\left[\int_{\left[m^{N},+\infty\right)} \omega(t)^{-\frac{1}{p-1}} d t\right]^{\frac{1}{p^{\prime}}}\left[\int_{\left[0, m^{N}\right]} \omega(t) d t\right]^{\frac{1}{p}}\left\|f_{2}\right\|_{L^{p}\left(\mathbb{R}^{+}, \omega\right) .}
\end{aligned}
$$

Using that $\omega$ satisfies the condition $\mathcal{M}_{p}^{y}\left(\mathbb{R}^{+}\right)$we complete the proof.

It is easy to check that any function $\omega_{r}(x)=x^{r}$ if $r>p-1$ satisfies the condition $\mathcal{M}_{p}^{0}\left(\mathbb{R}^{+}\right)$and the condition $\mathcal{M}_{p}((0,+\infty))$.

Corollary 6.1. Let $1<p<\infty$ and let $\omega_{r}(x)=|x|^{r}$ if $r>p-1$. Then the system $H^{+}(m)$ is an unconditional basis in the weighted norm space $L^{p}\left(\mathbb{R}^{+}, \omega_{r}\right)$. 
Theorem 6.2. For any $m=2,3, \ldots$ the system $H(m)$ is an unconditional basis in the weighted norm space $L^{p}(\mathbb{R}, \omega), 1<p<\infty$ if and only if there exist two points $y_{1} \in[0,+\infty], y_{2} \in[-\infty, 0]$ such that:

If $y_{1} \neq+\infty$ then $\omega$ satisfies the condition $\mathcal{M}_{p}\left(\mathbb{R}^{+} \backslash\left\{y_{1}\right\}\right)$ and the condition $\mathcal{M}_{p}^{y_{1}}\left(\mathbb{R}^{+}\right)$;

If $y_{1}=+\infty$ then $\omega$ satisfies the condition $\mathcal{M}_{p}\left(\mathbb{R}^{+}\right)$;

If $y_{2} \neq+\infty$ then $\omega$ satisfies the condition $\mathcal{M}_{p}\left(\mathbb{R}^{-} \backslash\left\{y_{2}\right\}\right)$ and the condition $\mathcal{M}_{p}^{y_{2}}\left(\mathbb{R}^{-}\right)$;

If $y_{2}=-\infty$ then $\omega$ satisfies the condition $\mathcal{M}_{p}\left(\mathbb{R}^{-}\right)$;

Corollary 6.2. Let $1<p<\infty$ and let $\omega_{r}(x)=|x|^{r}$ if $r>p-1$. Then the system $H(m)$ is an unconditional basis in the weighted norm space $L^{p}\left(\mathbb{R}, \omega_{r}\right)$.

\section{References}

[1] P. Cifuentes, K.S. Kazarian, A. San-Antolín; Characterization of scaling functions in a Multiresolution Analysis, Proc. of Amer. Math. Soc., 133 (2005), 1013-1022.

[2] P. Cifuentes, K.S. Kazarian, A. San-Antolín; Characterization of scaling functions, Wavelets and splines: Athens 2005, 152-163, Mod. Methods Math., Nashboro Press, Brentwood, TN, 2006.

[3] R.R. Coifman, C. Fefferman; Weighted norm inequalities for maximal functiona and singular integrals, Studia Math., 51 (1974), 241-250.

[4] I. Daubechies; Ten lectures on wavelets, SIAM, Philadelphia, 1992.

[5] J. Garcia-Cuerva, K.S. Kazarian, Calderón-Zygmund operators and unconditional bases of weighted Hardy spaces, Studia Math., 109 (1994), $255-276$.

[6] J. Garcia-Cuerva, K.S. Kazarian, Spline wavelet bases of weighted $L^{p}$ spaces, Proceedings of the American Mathematical Society, 123, 2 (1995), 433-439.

[7] J. Garcia-Cuerva and K. S. Kazarian, Spline wavelet bases of weighted spaces, in: Fourier Analysis and Part. Diff. Eq., Studies in Advanced Mathematics, CRC Press (1995), 169-184. 
[8] B.I. Golubov, Series in the Haar system, Itogi Nauki. Ser. Matematika. Mat. Anal. 1970 (1971), 109-146; trans. J. Soviet Math., 1:6 (1973), 704-726.

[9] G.Gripenberg, Necessary and sufficient conditions for the existence of a father wavelet, Studia Math., 114 (1995), 207-226.

[10] R.F. Gundy, R.L. Wheeden, Weighted integral inequalities for the nontangential maximal function, Lusin area integral and Walsh-Paley series, Studia Math., 49 (1974), 107-124.

[11] G.H. Hardy, J.E. Littlewood and G. Pólya, Inequalities, Cambridge University Press (1952).

[12] E. Hernández, G. Weiss; A first course on Wavelets, CRC Press, Inc. 1996.

[13] E. Hernández, X. Wang, G. Weiss; Characterization of wavelets, scaling functions and wavelets associated with multiresolution analyses, Function spaces, interpolation spaces, and related topics (Haifa, 1995), 51-87, Israel Math. Conf. Proc., 13, Bar-Ilan Univ., Ramat Gan, 1999.

[14] J.-P. Kahane; Some random series of functions, Cambridge University Press, 1993.

[15] K.S. Kazarian, The multiplicative completion of basic sequences in $L^{p}, 1 \leq p<\infty$ to bases in $L^{p}$, Dokl. Akad. Nauk Arm. SSR, 62 (1976), 203-209, (Russian).

[16] K.S. Kazarian, On the multiplicative completion of some incomplete orthonormal systems to bases in $L^{p}, 1 \leq p<\infty$, Analysis. Math., 4 (1978), 37-52, (Russian).

[17] K.S. Kazarian, On bases and unconditional bases in the spaces $L^{p}(d \mu)$, $1 \leq p<\infty$, Studia Mathematica, 71 (1982). 227-249.

[18] K.S. Kazarian, Summability of generalized Fourier series and Dirichlet's problem in $L^{p}(d \mu)$ and weighted $H^{p}$-spaces $(p>1)$, Analysis. Math., 13, 3 (1987), 173-197.

[19] K.S. Kazarian, The multiplicative completion of certain systems, Izv. Akad. Nauk Armyan. SSR, Ser. Mat., 13 (1978), 315-351, (Russian). 
[20] T. Kopaliani, Higher rank Haar wavelet bases in spaces $L_{w}^{p}(\mathbb{R})$, Georgian Math. J., 18, (2011), 517-532 .

[21] S.V. Kozyrev, Wavelet theory as $p$-adic spectral analysis, Izv. Math. 66:2 (2002), 367-376.

[22] A.S. Krantsberg, On the basisity of the Haar system in weighted spaces, Mosk. Inst. Elektr. Mat. 24 (1971).

[23] P. G. Lemarié-Rieusset, Ondelettes et poids de Muckenhoupt, Studia Mathematica, 108, no. 2 (1994), 127-147.

[24] J. Lindenstrauss, L. Tzafriri, Classical Banach spaces, v.1, Springer, 1977.

[25] S. Mallat, Multiresolution approximations and wavelet orthonormal bases for $L^{2}(R)$, Trans. of Amer. Math. Soc., 315 (1989), 69-87.

[26] J. Schauder, Eine eigenschaft des Haarschen orthogonalsystems, Math. Z., 28 (1928), 317-320.

[27] E.M. Stein, Singular integrals and differentiability properties of functions, Princeton, 1970.

[28] A. Torchinsky, Real-variable methods in harmonic analysis, Acad. Press, 1986.

[29] C. Watari, Mean convergence of Walsh Fourier series, Tôhoku Math. J., 16:2 (1964), 183-188.

[30] P.Wojtaszczyk, A mathematical introduction to wavelets, London Mathematical Society, Student Texts 37 (1997).

[31] S. Yano, On a lemma of Marcinkiewicz and its applications to Fourier series, Tôhoku Math. J., 11, (1959), 191-215.

[32] A. Zygmund, Trigonometric series, v. 1-2, Cambridge Univ. Press, 1959. 